\title{
Saccade Target Selection in the Superior Colliculus: A Signal Detection Theory Approach
}

\author{
Byounghoon $\mathrm{Kim}^{1}$ and Michele A. Basso ${ }^{1,2}$ \\ Departments of ${ }^{1}$ Physiology and ${ }^{2}$ Ophthalmology and Visual Sciences, University of Wisconsin-Madison, School of Medicine and Public Health, Madison, \\ Wisconsin 53706
}

\begin{abstract}
How the brain selects one action from among multiple options is unknown. A main tenet of signal detection theory (SDT) is that sensory stimuli are represented as noisy information channels. Therefore, the accuracy of selection might be predicted by how well neuronal activity representing alternatives can be distinguished. Here, we apply an SDT framework to a motor system by recording from superior colliculus (SC) neurons during performance of a color, oddball selection task. We recorded from sets of four neurons simultaneously, each of the four representing one of the four possible targets. Because the electrode placement constrained the position of the stimuli in the visual field, the stimulus arrangement varied across experiments. This variability in stimulus arrangement led to variability in choices allowing us to explore the relationship between SC neuronal activity and performance accuracy. SC target neurons had higher levels of discharge than SC distractor neurons in subsets of trials when selection performance was very accurate. In subsets of trials when performance was poor, the discharge level decreased in target neurons and increased in distractor neurons. Accurate performance was associated with larger separations between neuronal activity from targets and distractors as quantified by the receiver operating characteristic (ROC) area and $d^{\prime}$ (an index of discriminability). Poorer performance was associated with less separation of target and distractor neuronal activity. ROC area and $d^{\prime}$ scaled approximately linearly with performance accuracy. Furthermore, ROC area and $d^{\prime}$ increased as saccade onset approached. Together, the results indicate that SC buildup neuronal activity signals the saccadic eye movement decision.
\end{abstract}

Key words: saccade; eye movement; motor control; decision; population coding; multiple neuron recording

\section{Introduction}

A challenge to motor systems is the need to select a single action from among multiple options. Evidence implicates the superior colliculus (SC), a topographically organized map of rapid eye movements (saccades) located within the midbrain, in the process of selection for action (Apter, 1945; Robinson, 1972; Basso and Wurtz, 1997, 1998; Horwitz and Newsome, 1999, 2001; Krauzlis and Dill, 2002; McPeek and Keller, 2002, 2004; Carello and Krauzlis, 2004), although exactly how SC contributes to selection for action is unknown. Traditionally, experiments in SC were performed with single spots of light as targets for saccades. More recently, recordings of SC neurons occur in the presence of more complex stimulus displays that include targets and distractors (Ottes et al., 1987; Glimcher and Sparks, 1993; Basso and Wurtz, 1998; Edelman and Keller, 1998; McPeek and Keller, 2002; Port and Wurtz, 2003). The stimulus displays are sometimes adopted from those used in psychophysical experiments

Received Dec. 7, 2007; revised Jan. 5, 2008; accepted Jan. 15, 2008.

This work was supported by National Institutes of Health Grant EY13692 (M.A.B.). We also acknowledge the support of National Center for Research Resources Grant P51 RR000167 to the Wisconsin National Primate Research Center.

Correspondence should be addressed to Dr. Michele A. Basso, Department of Physiology, University of Wisconsin-Madison, School of Medicine and Public Health, 1300 University Avenue, Room 127 SMI, Madison, WI 53706. E-mail: michele@physiology.wisc.edu.

DOI:10.1523/JNEUROSCI.5424-07.2008

Copyright $\odot 2008$ Society for Neuroscience $\quad$ 0270-6474/08/282991-17\$15.00/0 designed to explore visual search (Treisman and Gelade, 1980; Egeth and Yantis, 1997; Schall and Thompson, 1999; Palmer et al., 2000; Wolfe and Horowitz, 2004). In the simplest arrangement, an array of visual stimuli appears and one member of the array differs along a single feature dimension such as color or orientation. The task is to select the oddball stimulus and make a saccadic eye movement to it. Analyses based in signal detection theory (SDT) (Green and Swets, 1966) combined with recordings of neurons made one at a time, are used to determine whether and at what time neurons discriminate between targets and distractors (Schall et al., 1995; Thompson et al., 1996; McPeek and Keller, 2002; Thomas and Paré, 2007). The results of these experiments indicate that saccade target selection likely results from a distributed network of activity across populations of neurons within at least frontal eye field (FEF), lateral intraparietal area (LIP), and SC (Hanes and Schall, 1996; Thompson et al., 1996; Schall and Hanes, 1998; Schall and Thompson, 1999; Hanes and Wurtz, 2001; Paré and Wurtz, 2001; McPeek and Keller, 2002; Thomas and Paré, 2007).

In this study, we apply an SDT approach to further our understanding of how the SC contributes to selection. Our approach was different from that used previously in which SDT was used as a statistical tool to determine when neurons distinguish the presence of a target in their response field (RF) relative to saccade latency (McPeek and Keller, 2002). Our approach was more similar to that used in extrastriate cortical areas to explore 
the relationship between neuronal activity and perceptual accuracy (Britten et al., 1992; Parker and Newsome, 1998; Dodd et al., 2001). In this latter approach, individual trials are sorted based on performance accuracy and receiver operating characteristic (ROC) curves are computed to determine how well an ideal observer could predict the behavioral choice based on the activity of the neurons (Britten et al., 1992, 1996; Shadlen et al., 1996; Parker and Newsome, 1998; Dodd et al., 2001; Krug et al., 2004). In both applications of SDT, neurons are recorded one at a time and the responses of neurons to the preferred stimulus (or when the stimulus is a target) are compared with responses of the same neurons to a nonpreferred stimulus (or when the stimulus is a distractor). This comparison is referred to as the neuron, anti-neuron assumption (Britten et al., 1992).

Here we focused on the relationship between SC neuronal activity and selection accuracy. Although in the latter part of this study we also address the timing of selection. Four stimuli appeared in the visual field. Importantly, we recorded from sets of four neurons simultaneously: one neuron representing each of the four SC neuronal populations underlying the selec-

tion. Therefore, we did not need the neuron, anti-neuron assumption. By performing ROC analysis and computing $d^{\prime}$, an index of discriminability, we found that the separation and discriminability of the neuronal activity representing targets and distractors scaled with performance accuracy. The scaling of discriminability with performance accuracy occurred in the neuronal activity measured even $20 \mathrm{~ms}$ before the onset of a saccade. Together, the results provide evidence that buildup neuron activity signals a decision variable for saccadic eye movements.

\section{Materials and Methods}

Physiological and eye movement monitoring procedures. For electrophysiological recording of SC neurons and monitoring eye movements, cylinders, and eye loops were implanted in three rhesus monkeys (Macaca mulatta) using documented procedures (Judge et al., 1980; Li and Basso, 2005). We recorded from 176 neurons within the intermediate layers of the monkey SC. Neurons were recorded simultaneously in sets of four. In monkey $\mathrm{m}$, we recorded 13 sets $(n=52)$. In monkey c, we recorded 17 sets $(n=68)$. In monkey w, we recorded 14 sets $(n=56)$. Of the total 176 neurons in three monkeys, all neurons were defined statistically as buildup/prelude (McPeek and Keller, 2002; Li and Basso, 2005), except six, which were defined statistically as visual-tonic (McPeek and Keller, 2002; $\mathrm{Li}$ and Basso, 2005). Neurons were recorded with four independently moveable, tungsten microelectrodes (FHC, Bowdoin, ME) with impedances between 0.3 and $1.0 \mathrm{M} \Omega$ measured at $1 \mathrm{kHz}$. Four electrodes were aimed at the SC, each through different stainless-steel guide tubes held in place by a plastic grid secured to the cylinder (Crist et al., 1988). Two were aimed at one SC and two were aimed at the other SC. Action potential waveforms were filtered and amplified by a differential amplifier (Alpha Omega, Nazareth, Israel; MCP-Plus) and then sampled and digitized (Measurement Computing, Norton, MA; PCI-DAS4020/16). The digitized waveforms were identified and sorted with an interactive computer program (Mex) allowing the experimenter to sort waveforms in real time. For two of the three monkeys, neuronal data were also saved to disk

\section{task}

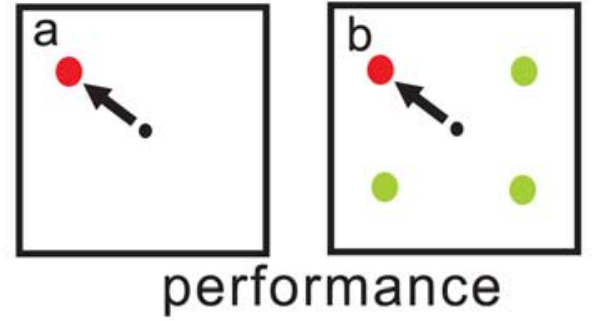

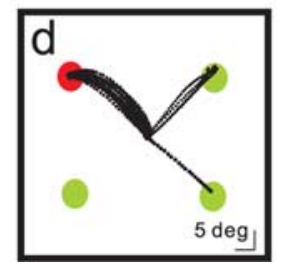

$>75 \%$
$<75 \%$

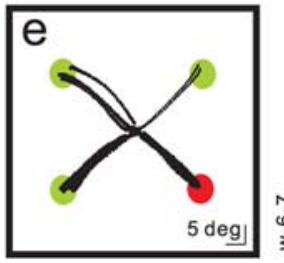

$0 \%$

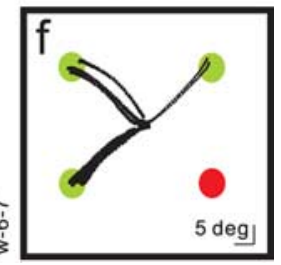

Figure 1. Target selection task. Each square shows the spatial arrangement of the task. Red filled circles are targets and green filled circles are distractors. The required saccade is indicated schematically by black arrows and actual saccade trajectories are spot disappeared simultaneously. $\boldsymbol{a}$, The monkeys' task was to make a saccade to the spot presented alone in the visual field or to made with $<75 \%$ accuracy. Many saccades were made to the distractors (green circles). $\boldsymbol{f}$, A subset of the trials shown in $\boldsymbol{e}$ when te that the target and distractor positions are normalized to 45, 135, 225, and 315 positions.

as waveforms and sorted off-line to confirm the adequacy of the on-line discrimination. For this we used custom software (written and compiled in Delphi 5.0) that sorted spikes based on time-voltage criteria.

Using the magnetic induction technique (Fuchs and Robinson, 1966) (C.N.C. Engineering, Seattle, WA), voltage signals proportional to horizontal and vertical components of eye position were filtered (8 pole Bessel $-3 \mathrm{~dB}, 180 \mathrm{~Hz})$, digitized at 16-bit resolution and sampled at 1 $\mathrm{kHz}$ (National Instruments, Austin, TX; PCI-6036E). The data were saved for off-line analysis using an interactive computer program (Dex) designed to display and measure eye position and calculate eye velocity. We used an automated procedure to define saccadic eye movements by applying velocity and acceleration criteria of $50 \%$ and $5000 \% \mathrm{~s}^{2}$, respectively. The adequacy of the algorithm was verified on a trial-by-trial basis by the experimenter.

All experimental protocols were approved by the University of Wisconsin, Madison, Institutional Animal Care and Use Committee and complied with and generally exceeded the standards set by the Public Health Service policy on the humane care and use of laboratory animals.

Behavioral procedures and task. We used a real-time experimental data acquisition and visual stimulus generation system (Rex, Vex and Mex, developed and distributed by National Institutes of Health) (Hays et al., 1982) to create the behavioral paradigms and acquire two channels of eye position and four channels of neuronal data. Trained monkeys sat in a custom designed primate chair with head stabilized during the experimental session (typically 3-5 h). Visual stimuli were rear-projected onto a screen at $51 \mathrm{~cm}$ distance using a projector (LP130; Infocus, Wilsonville, OR) with a native resolution of $1024 \times 768$ and operating at $60 \mathrm{~Hz}$. A photocell secured to the screen sent a transistor-transistor logic pulse to the experimental personal computer (PC) providing an accurate measure of stimulus onset. The fixation spot at the center of the screen had a (mean of three measurements) luminance of $1.52 \mathrm{~cd} / \mathrm{m}^{2}$. Visual stimuli each had luminance values of $5.8 \mathrm{~cd} / \mathrm{m}^{2 \mathrm{~m}}$ (mean of three measurements each). The background luminance was $0.58 \mathrm{~cd} / \mathrm{m}^{2}$ (mean of three measurements). The PC for the visual stimulus display was a slave device to the PC used for experimental control and data acquisition. 

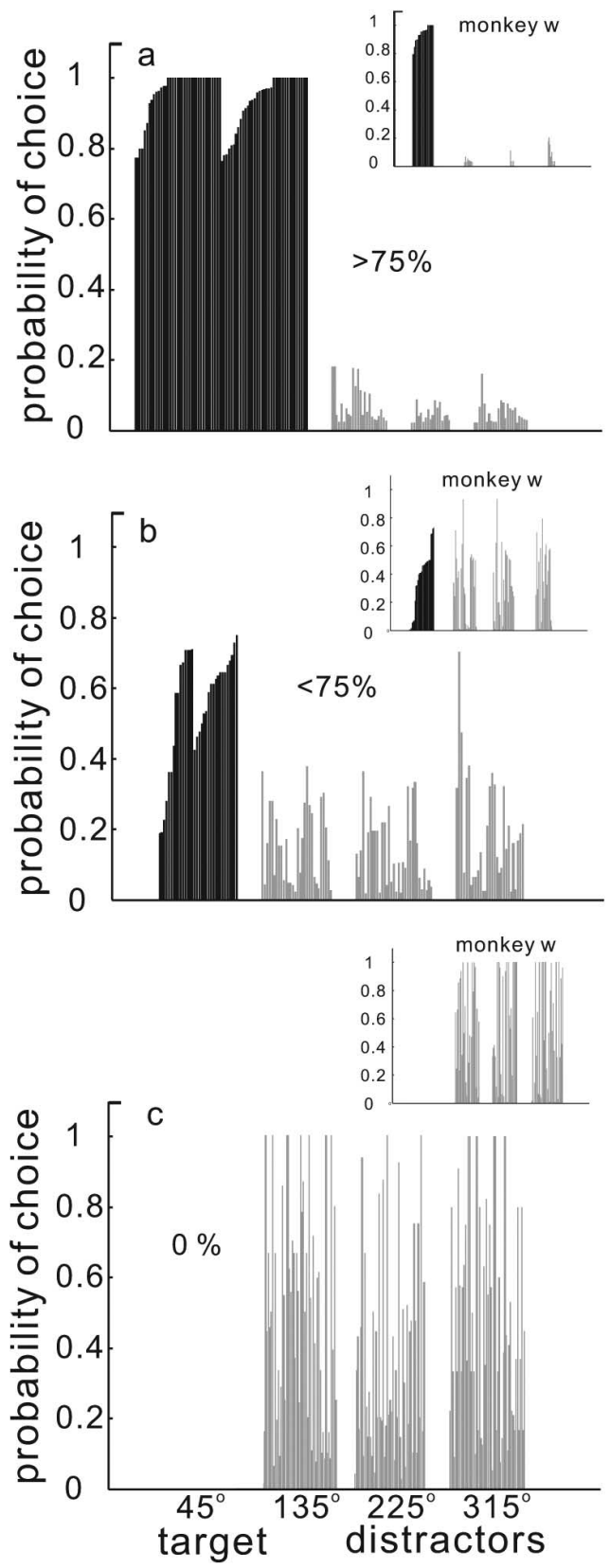

Figure 2. Performance in the selection task. The probability of a saccade choice to the targets $\left(45^{\circ}\right.$, black bars) or the distractors $\left(135^{\circ}, 225^{\circ}, 315^{\circ}\right.$, gray bars) is plotted against the normalized position of the stimuli in the array. $\boldsymbol{a}$, Data from two monkeys (monkeys $\mathrm{c}$ and $\mathrm{m}$ ) selected for performance $>75 \%$ correct. $\boldsymbol{b}$, Data from two monkeys selected for $<75 \%$ correct performance. c, Subset of the data from the monkeys on error trials (0\%). The insets in all panels show data from monkey w. We separated monkey w's data because of its poorer performance.

After fixating on a centrally located spot $\left(0.2^{\circ}\right.$ diameter $)$ for a random time of $1800-2300 \mathrm{~ms}$, four spots $\left(0.5^{\circ}\right.$ diameter $)$ appeared and the central spot disappeared. Each spot was located in the center of each empirically defined RF of the four SC neurons (see Fig. 1a). The task required monkeys to choose the differently colored target within $\sim 300$ $\mathrm{ms}$ by making a saccade to the differently colored spot immediately after the disappearance of the fixation spot (simultaneous with the array onset). The target could be either red among green distractors or green among red distractors. The color arrangement of the display was fixed each day of recording but varied across recording days. After making a choice, monkeys maintained fixation at the target spot for a random time of 500-600 ms and then received fluid reward. The location of the target spot was randomized among the four possible locations. On interleaved trials, a single spot appeared in each of the four possible locations (see Fig. $1 a, b)$. Two spots appeared in each hemifield although the exact location of the visual spots depended on the location of the four electrodes within the SC. Further details are provided below.

Data analysis. All statistical analyses were performed using Matlab (MathWorks, Natick, MA). We performed statistical comparisons when appropriate using the nonparametric test of median differences, Wilcoxon rank sum (Keppel, 1991). We computed ROC curves based on SDT (Green and Swets, 1966; Cohn et al., 1975; Bradley et al., 1987; Britten et al., 1992; Thompson et al., 1996). For each trial, we convolved each spike in a spike train with a Gaussian having a $\sigma=4 \mathrm{~ms}$ (McPeek and Keller, 2002), although the data are displayed in the figures with a 10 ms Gaussian kernel. We divided neurons from each data set by performance (percentage of correct trials for each target position) into different groups. One was $>75 \%$ and $<75 \%$ accuracy rates (see Fig. 6); a second was $35,65,75,85$, and $100 \%$ accuracy rates (see Fig. 7); and a third was correct trials versus error trials in which the same saccade was made (see Fig. 12). Data were also sorted by target distance from the fixation point (see Fig. $8 a$ ) and saccade velocity (see Fig. $8 b$ ). To compute ROC curves, we computed the probability that the discharge rate exceeded a criterion in each measurement epoch on a trial-by-trial basis for each neuron. The criterion was incremented from the minimum to the maximum discharge rate in the epoch in step sizes of (maximum-minimum discharge rate/100). A probability value was computed for each criterion. A single point on the ROC curve was produced for each increment in the criterion and the entire ROC curve was generated from all the criteria. The area under the ROC curve measures the separation between the two distributions (target and maximum distractor neuronal activity) and provides a measure of the probability that a random draw from each of the two distributions would yield a value that is larger for the target than for the distractor when the monkey correctly selects the target. An ROC area of 0.50 indicates that the two distributions overlap completely. To quantify the discriminability of the target and distractor neuronal activity distributions, we computed $d^{\prime}$, the ratio of the differences between the means of the distributions to the sum of the SDs of the distributions $d^{\prime}=$ $\left(\mu_{\text {target }}-\mu_{\text {distractor }}\right) / \sigma_{\text {target }}+\sigma_{\text {distractor. }}$ Unless explicitly stated (see Figs. $9-11)$, we computed the mean discharge rate during the $100 \mathrm{~ms}$ time interval preceding saccade onset for the target neuronal activity. The distractor with the highest discharge rate during the same interval was determined on each trial. In so doing, the distractor neurons contributing to the ROC could vary randomly on a trial-to-trial basis ensuring the application of a max rule for every trial. For the time course analysis shown in Figure 11, we performed the ROC analysis as described above but across $1 \mathrm{~ms}$ intervals, forward in time beginning at the onset of the stimulus array and ending at saccade onset. Whereas for the stationary ROC analysis we selected the distractor activity with the maximum discharge rate measured $100 \mathrm{~ms}$ before saccade onset (or in $20 \mathrm{~ms}$ epochs as described in the text), for the time course analysis shown in Figure 11, the distractor neuron activity used for analysis was determined dynamically by selecting the distractor with the largest activity (spikes per second) at each millisecond interval of each trial.

\section{Results}

Trained monkeys made saccades in a task requiring the selection of a single red target from an array of one red and three green stimuli (or vice versa) (Fig. 1a). We recorded from four neurons simultaneously ( $n=44$ sets of four) from three monkeys using independently movable electrodes. The preferred saccade and the class of neuron were assessed empirically and statistically using a delayed-saccade task with a single target (Li and Basso, 2005; Li et al., 2006). The neurons contributing to this analysis were defined as buildup/prelude neurons (see Materials and Methods) (Munoz and Wurtz, 1995; Basso and Wurtz, 1998; McPeek and Keller, 2002). For the selection task, we arranged the array of stimuli so that each stimulus fell within the center of one RF of each of the four neurons. Because the exact position of the stimuli depended on the positioning of each of the four electrodes within the SC, 
the stimulus positions were not always perfectly symmetric within the visual field. Although for all experiments, two stimuli were always located in each hemifield and we ensured no overlap of any of the RFs. For analyses and clarity of display, we normalized the stimulus positions to $45,135,225$, and $315^{\circ}$.

\section{Selection performance is variable}

When a single stimulus appeared in the visual field, monkeys made saccades to the correct target location invariably, not surprisingly (Fig. 1a,c). In contrast, when an array of stimuli appeared, monkeys made saccades as quickly to the differently colored target but sometimes made errors and selected the wrong stimulus (Fig. 1b,d-f). In general, the monkey selected the correct target with $>75 \%$ accuracy (Fig. $1 d$ ). For some target positions or on some experimental days, however, performance was less accurate $(<75 \%)$ (Fig. 1e). For the example set of data shown in Figure 1, when the target was located at the $315^{\circ}$ position, the monkey sometimes made saccades to one of the green stimuli even though the red stimulus was the target. When the monkey selected a green stimulus it did not receive a reward. In some cases, the monkey made mistakes on every trial and failed to select the target altogether, choosing a distractor stimulus instead (Fig. $1 f$ ). These trials also were not rewarded. Figure 1 shows just one example data set from one monkey. Across our three monkeys the positions of the target varied within experimental sessions. Whether there was a green target among red distractors or a red target among green distractors varied across experimental days.

As indicated above, the positions of the stimuli in the display were constrained by the positions of the electrodes in the two SCs. This led to asymmetric visual displays and as a result, variability in the monkeys' performance. This turned out to be useful, because it meant that we could explore the relationship between neuronal activity and variations in choice accuracy even when the sensory information determining the choice (a color discrimination) remained the same. This tack is similar to that used when choice accuracy is compared with neuronal activity during perceptual reports of bistable images (Dodd et al., 2001; Parker et al., 2002) or in a random dot motion discrimination task when the display has 0\% coherence (Newsome et al., 1989a,b; Britten et al., 1992, 1996). We first describe the behavioral performance of our monkeys. Then we show examples of activity from target and distractor neurons in the task. We then present the SDT analysis results.

We sorted the 44 data sets based on performance into three arbitrary groups, $>75,<75$, and $0 \%$ accuracy. Overall, two of our three monkeys performed the task similarly and reasonably well (monkeys $\mathrm{c}$ and $\mathrm{m}$ ), whereas monkey w performed the task particularly poorly. The data for monkey $\mathrm{c}$ and $\mathrm{m}$ were accumulated from 26 recording sessions. For the analysis described below, there were 3199 correct trials and 1054 errors trials from these two monkeys. Because monkey w performed the task less well than the other two monkeys, we present the data from monkey w independently of the data from monkey $\mathrm{c}$ and $\mathrm{m}$ throughout. The data from monkey w were accumulated from 14 recording sessions and include 1391 correct trials and 2862 error trials. Figure 2 shows the performance across all experimental sessions and all three monkeys in the selection task. Figure $2 a$ shows the distribution of the monkeys' choices to the target or the distractors when performance was good $(>75 \%)$. For two monkeys, the majority of trials were performed correctly and the monkeys made saccades to the target (Fig. 2a, black bars). Although in some cases, they performed less well and made saccades to the distractors instead of the target (Fig. $2 b$, gray bars). On a minority of the

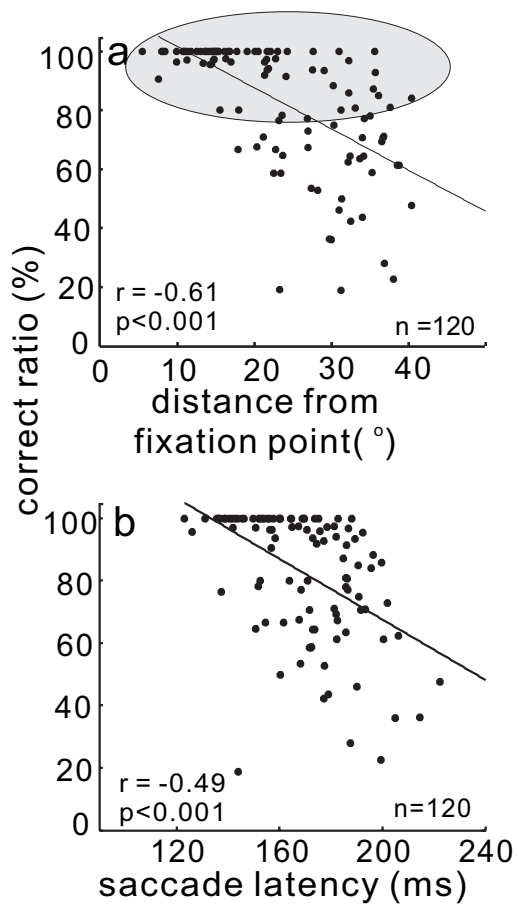

Figure 3. Saccade choices vary with target locations in the selection task. $\boldsymbol{a}$, The ratio of correct to total trials expressed as a percentage is plotted against the amplitude of the target position relative to the fixation point in degrees. The amplitude of the target position was computed as target distance (in degrees) $=\sqrt{x^{2}+y^{2}}$, where $x$ was the horizontal position of the target and $y$ was the vertical position of the target. The fixation point was located at Cartesian coordinate 0,0 . Each dot shows the target position for each experimental session from two monkeys (monkey $\mathrm{m}$ and monkey $\mathrm{c}$ ). Despite an overall correlation between performance accuracy and distance of the target from the fixation point, there was a broad range of amplitudes associated with $\sim 80 \%$ accuracy as indicated schematically by the gray ellipse. $\boldsymbol{b}$, Ratio of correct to total trials (percentage) is plotted against saccade latency. As the percentage of correct choices increased, saccade latency decreased. The solid black line is the best fit linear regression. Each dot is from one neuron when it had the target in its RF. The total number of neurons ( $n$ ) was 120 (30 sets of 4 from two monkeys). $r$ is the Pearson $r$ value.

trials, monkeys made errors and looked only at the distractors (Fig. 2c, gray bars). The behavior of one monkey (monkey w) was poorer than the other two (Fig. $2 a-c$, insets). Although this monkey chose each of the distractor locations with equal probability (Fig. 2c, inset), it overall made more errors than the other two monkeys (Fig. 2, compare $a, b$, insets).

Because it is known that increasing the distance between targets and distractors can influence performance (Meinecke, 1989; Wolfe and O'Neill, 1998; Motter and Simoni, 2007), we reasoned that the variability in the monkeys' selection accuracy may result from differences in the location of the target relative to the fovea and relative to the other distractors. Because our stimulus configurations were constrained by the sites of electrode penetrations, we considered this possibility. Note, however, that two stimuli were located in one hemifield and two were located in the opposite hemifield for all cases. Nevertheless, as it turned out, monkeys tended to make more errors because of the position of the stimuli within the array relative to the fixation point. We found that as the distance of the target from the fovea increased, performance accuracy decreased (Fig. $3 a$ ). There was a statistically significant correlation between the ratio of correct to total trials performed and the distance of the target from the fixation point $(r=-0.61, p<0.001)$ as measured by target distance (in degrees $)=\sqrt{x^{2}+y^{2}}$, where $x$ was the horizontal position of the target and $y$ was the vertical position of the target and the fixation 

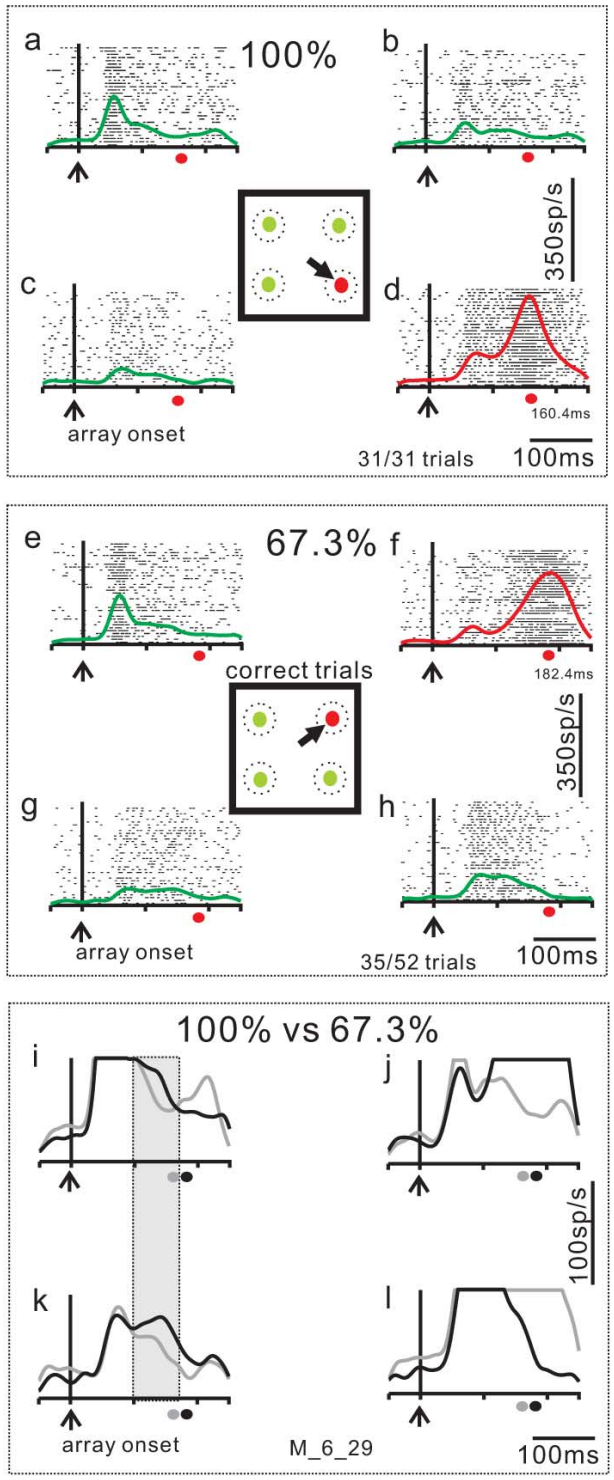

Figure 4. Example of target and distractor neuronal activity in the selection task. An example set of four neurons recorded simultaneously during performance of the selection task. $\boldsymbol{a}-\boldsymbol{c}$ $\mathrm{SC}$ neuronal activity recorded in the target selection task when distractors were located in the RF of the recorded neuron (distractor neurons). $\boldsymbol{d}, \mathrm{SC}$ neuronal activity when the target was located in the neuron's RF (target neuron). In each panel, each tick is an action potential and each row of ticks is one trial. The envelope of activity (SDF, $\sigma=10 \mathrm{~ms}$ ) is superimposed on the rasters. Red SDFs are from target neurons and green SDFs are from the distractor neurons. Each of the four panels is aligned on the onset of the array of spots indicated by the vertical line and the upward arrow (array onset). The square in the center of the four panels show the stimulus display. The black arrow indicates the correct choice of saccade to the differently colored target. The dashed circles are schematics of neuronal RFs to emphasize the fact that we recorded from four neurons simultaneously. The trials shown in this figure are sorted based on performance. $\boldsymbol{a}-\boldsymbol{d}$ show the neuronal activity when the monkey accurately performed all trials with the target located at the $315^{\circ}$ position ( 31 of 31 trials, 100\%). The filled red circle below each panel marks the average saccade latency made to the correct target for reference. $\boldsymbol{e}-\boldsymbol{h}$ show the subset of trials in which the monkey chose the saccade target correctly when it appeared at the $45^{\circ}$ position. The monkey performed the $45^{\circ}$ position trials less well ( 35 of 52 trials; $67.3 \%$ correct) compared with the $315^{\circ}$ position trials. $\boldsymbol{i}-I$ directly compare the SDFs for the trials in which the monkey performed the $315^{\circ}$ target with $100 \%$ accuracy and the trials in which the monkey performed the $455^{\circ}$ target with $67.3 \%$ accuracy. The gray traces are the same as those shown in $\boldsymbol{a}-\boldsymbol{d}$. The black traces are the same as those shown in $\boldsymbol{e}-\boldsymbol{h}$. Note the expanded vertical scale. The gray filled circle on the abscissa is the saccade latency on the $100 \%$ correct trials. The black filled circle on the abscissa is the saccade latency on the $67.3 \%$ correct trials. The shaded great rectangle shows that the distractor neuronal activity was different in these two subsets of trials. The $M \_6 \_29$ indicates the data come from monkey $m$ and the date, $6 \_29$ was used as a file identifier. point was located at Cartesian coordinate 0,0 . We also found that as the correct ratio increased, saccade latency decreased (Fig. $3 b$ ). This result suggests that the monkeys found the task easier when the target was closer to the fixation point, although we did not explicitly manipulate variables to make the task easier for the monkeys (Bichot and Schall, 2002). The relationship between the correct ratio and saccade latency was statistically significant $(r=$ $-0.49, p<0.001)$. The distance of the target from the fixation point, however, was not the sole factor responsible for variations in choice accuracy because monkeys were able to perform with a high level of accuracy for all target distances on at least some of the trials. This is indicated schematically by the gray ellipse around the data shown in Figure $3 a$.

In light of these behavioral observations, we reasoned that we could capitalize on the variation in behavioral performance to provide insight toward understanding the relationship between neuronal activity in SC and saccade choice accuracy. Below we show that applying an SDT approach reveals insights into how SC neuronal activity predicts saccade choice in the oddball selection task.

\section{Saccade target selection and SC neuronal population activity}

Figure 4 shows one example from four SC neurons recorded simultaneously. Each neuron had a single stimulus within its RF and, therefore, each neuron had a discharge of action potentials associated with the onset of the visual stimulus. After the initial visual response, the activity of the neurons representing the distractors decreased over time (Fig. $4 a-c$, green traces) and the activity of the neuron representing the target increased (Fig. $4 d$ ). Ultimately, the target neuron showed the characteristic saccaderelated burst of activity (Wurtz and Goldberg, 1972; Sparks, 1975) (Fig. $4 d$, red traces). For all of the trials shown in Figure $4 a-d$, the monkey made the correct saccade to the target located in the $315^{\circ}$ position ( 31 of 31 trials). The mean saccade latency for these target trials was $160.40 \mathrm{~ms}$ and is indicated in Figure $4 a-d$ on the abscissa by the filled red circle.

For each experimental session, the target positions were randomized among the four possible locations. An example of the recording from the same four SC neurons during performance of the subset of trials in which the target was located in the $45^{\circ}$ position is shown in Figure $4 e-h$. For this target position the monkey performed with $67.3 \%$ accuracy. The monkey made the correct saccade on 35 of 52 trials. The mean saccade latency on correct target trials was $182.40 \mathrm{~ms}$, indicated on the abscissa in Figure $4 e-h$ by the filled red circle. Despite the fact that these trials were also correct, the mean saccade latency was longer for this target position than for saccades made to the $315^{\circ}$ position. The difference between the saccade latency in the $100 \%$ accuracy subset of trials (160.40 ms) and the saccade latency in the $67.3 \%$ accuracy subset of trials $(182.40 \mathrm{~ms})$ was statistically significant (Wilcoxon rank sum, $p<0.01$ ). It is unclear what exactly caused the monkey's poor choices or the increase in latency in the $45^{\circ}$ position trials compared with the $315^{\circ}$ position trials but the observation is consistent with an increase in difficulty on these trials. What might cause the increase in difficulty could be a change in the activity of the neurons representing the targets (Basso and Wurtz, 1997, 1998; Dorris and Munoz, 1998). But, perhaps also a change in the activity of neurons representing the distractors might contribute. Based on this, we hypothesized that the relative level of activity between the target and distractor neurons might be associated with the certainty monkeys had regarding the target and therefore, might be related to selection accuracy. 
To see whether changes occurred in distractor neuron activity when performance accuracy varied, we compared directly the neuronal activity for correct trials when the saccade was made to the $315^{\circ}$ target with the neuronal activity for correct trials when the saccade was made to the $45^{\circ}$ target (Fig. $4 i-l$ ). The Figure $4 i-l$ shows the spike density functions (SDFs) from the $100 \%$ correct trials and the $67.3 \%$ correct trials superimposed. The gray traces are the $100 \%$ trials shown in Figure $4 a-d$ and the black traces are the $67.3 \%$ trials shown in $e-h$. The vertical scale is expanded for a direct comparison between the distractor activities. The saccaderelated increase in activity associated with correct trials in the neuron coding the $45^{\circ}$ target (Fig. $4 j$, black trace) and the $315^{\circ}$ target (Fig. $4 l$, gray trace) appeared as expected, but is blunted because of the vertical scaling. The neuronal activity associated with the distractor positions, however, was different in these two subsets of trials. This was particularly evident for the distractor located down and to the left (Fig. $4 k$, compare gray and black traces). Note that the difference in the activity of these two neurons shown in Figure 4, $i$ and $k$, occurred despite the fact that the stimuli located in these positions were distractors in both subsets of trials (Fig. $4 i, k$, shaded rectangle). When the monkey performed the selection task with $100 \%$ accuracy, the activity of these distractor neurons was lower than when the monkey performed the selection task with 67.3\% accuracy (Fig. 4i,k, compare gray and black traces). This observation suggests two important things. First, SC neuronal discharge coding targets and distractors contributes to saccade selection in this task. Second, it is the relative level of activity between the target neurons and the distractor neurons that determines selection accuracy.

\section{Discriminability of target and distractor neuronal activity predicts performance}

To quantify the relationship between target and distractor neuronal activity and selection performance, we adopted an SDT approach to analyze the multiple neuron data. A basic premise of SDT is that sensory stimuli are represented as random variables that are noisy and independent from one another. Therefore, on each trial, each stimulus in the array ought to activate a population of neurons with a level of activity that varies about some mean level. To select the saccade target, the mean level of activity of neurons representing the target should be higher than the mean level of activity representing the distractor stimuli. Furthermore, the accuracy of the selection should be predicted by the disciminability between the target and distractor neuronal activity (Verghese, 2001). For example, when the distributions of neuronal activity representing the target and the distractors are widely separated, such as would occur with large mean differences in discharge rate, saccade choices should be highly accurate. In contrast, if the distributions of neuronal activity representing the target and distractors are highly overlapping, then selection accuracy should be poor. Therefore, we first measured the mean and SD of activity from target and distractor neurons across all SC neurons recorded in correct and error trials. After combining all of the data, we computed the probability of obtaining a particular discharge rate on a trial-by-trial basis for target and distractor neurons. We then sorted all the neurons using only the correct trials into two groups based on performance accuracy.

Figure 5 shows the probability distributions of neuronal activity measured in the $100 \mathrm{~ms}$ interval before saccade onset for the target neurons and the distractor neurons. The neuronal activity measured in the target neurons and the distractor neurons in trials when only a single stimulus appeared is shown in Figure $5 a$. As expected, when a single target appeared, the distractor activity
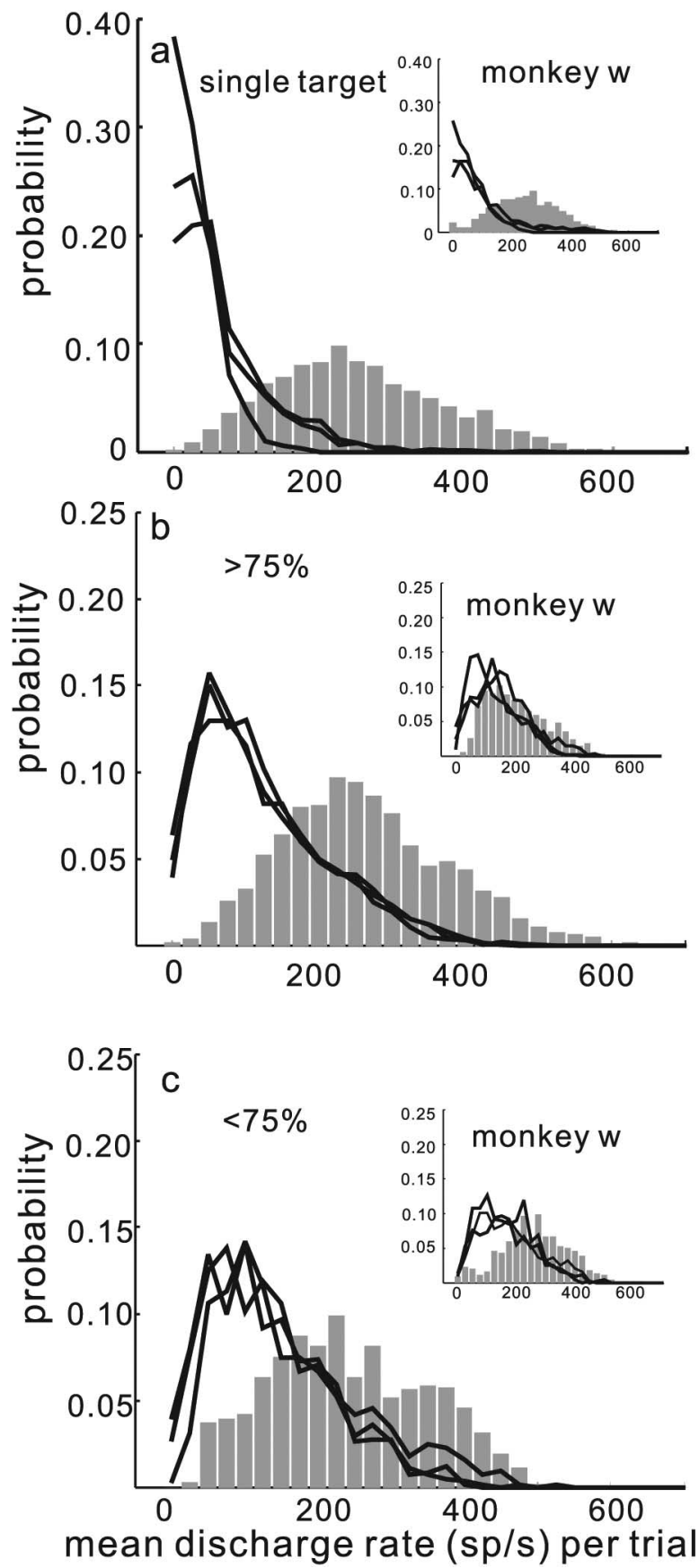

Figure 5. The separability of discharge rates between target and distractor neurons varies. The probability of measuring a particular discharge rate in target and distractor neurons is plotted from all of the data from all three monkeys. The data come from a total of 176 neurons and 4590 correct trials. The insets show the data for monkey w. $\boldsymbol{a}$, Probability is plotted against the mean discharge rate measured $100 \mathrm{~ms}$ before saccade onset from the data collected in the single target condition. The same interval, using the saccade latency to the target, was used to calculate the distractor neuron activity. The black lines show the probability distribution of discharge rates for each of the three distractor neurons in the single target condition. The gray bars show the probability distribution for the discharge rates measured from target neurons in the single target condition. $\boldsymbol{b}$, Probability is plotted against mean discharge rate measured on trials in which monkeys performed the four stimuli task with $>75 \%$ accuracy. The arrangement is the same as in $\boldsymbol{a}$. $\boldsymbol{c}$ shows the same as $\boldsymbol{b}$, but for trials in which monkeys performed with $<75 \%$ accuracy.

was low, with a mean discharge rate of 85.82 spikes/s and a SD of 65.86 spikes/s (Fig. $5 a$, black lines) across all the data from two monkeys ( $n=30$ sets of four; 120 neurons from monkey $\mathrm{m}$ and c). In contrast, the mean activity measured in the target neurons 
was much higher. We measured a mean discharge rate of 248.75 spikes/s and an SD of 112.93 spikes/s (Fig. 5a, gray bars).

The results obtained when four stimuli appeared and monkeys correctly selected the target with an accuracy $>75 \%$ is illustrated in Figure $5 b$. The mean discharge rate across all neurons from monkeys $\mathrm{c}$ and $\mathrm{m}$ ( $n=30$ sets of four; 120 neurons) measured from distractor neurons when monkeys performed the task with $>75 \%$ accuracy was 183.90 spikes/s (Fig. $5 b$, black lines). The SD of the distribution of discharge rates was 93.80 spikes/s. The mean discharge rate measured from target neurons when monkeys performed the task with $>75 \%$ was 262.67 spikes/s (Fig. $5 b$, gray bars). The SD of the distribution of discharge rates in the target neurons was 111.13 spikes/s. The median discharge rates of target and distractor neurons in the $>75 \%$ performance condition were significantly different (Wilcoxon test, $p<0.001$ ). In the subset of trials in which the monkeys performed less well $(<75 \%$ correct) the mean discharge rate across all distractor neurons was 217.13 spikes/s (Fig. $5 c$, black lines) whereas the mean discharge rate across all target neurons was 241.24 spikes/s (gray bars). The SD values of the distributions of discharge rates in the target and distractor neurons for the $<75 \%$ accurate subset, were 89.55 and 107.90 spikes/s, respectively. The median discharge rates between the target and distractor neurons in the $<75 \%$ correct trials were significantly different (Wilcoxon test, $p<0.001$ ). For both monkeys $\mathrm{c}$ and $\mathrm{m}$ there was a decrease in the neuronal activity associated with the target (262.07-241.24 spikes/s) and an increase in neuronal activity associated with the distractors (183.90-217.13 spikes/s) when performance changed from more to less accurate. The median differences between these two conditions were statistically significant (Wilcoxon test, $p<0.001$ ). Although the SD values for the target neuron activity decreased from 111.13 to 89.55 spikes/s when performance decreased, this difference was not statistically significant (Levene's test, $p=0.76$ ). In contrast, there was an increase in the SD of activity in the distractor neuron distributions when performance went from $>75$ to $<75 \%$ (93.80-107.90 spikes/s). This increase in SD was statistically significant (Levene's test, $p<0.01$ ).

These results show that the probability of obtaining a level of discharge in target and distractor neurons within the SC varies with performance accuracy. When performance accuracy is high the difference between the discharge of target and distractor neurons is high. When performance accuracy is low, the difference between the discharge of target and distractor neurons is low. Furthermore, the variability in discharge rate of target neurons is similar regardless of performance; however, the variability in distractor neuron activity increases as performance decreases. These results are consistent with the hypothesis that when the populations of neuronal activity representing the targets and distractors are highly discriminable monkeys are likely to perform well in the selection task. When the populations of target and distractor neuronal activity are less discriminable, performance will be poor. Therefore, we conclude that it is the relative level of target and distractor neuronal activity in buildup neurons of the SC that predicts performance accuracy. We quantify this using ROC analysis below.

In contrast to monkeys $\mathrm{c}$ and $\mathrm{m}$, monkey $\mathrm{w}$ performed the selection task overall less well (Fig. 2, insets). For this reason, we presented the data from monkey w separately. The insets in Figure 5 show the probability of discharge rates measured in target and distractor neurons sorted by performance for monkey w. When only a single target appeared, the mean and SD of the discharge rates across all neurons ( $n=14$ sets of 4 ; 56 neurons) for distractor neurons were 143.93 and 132.09 spikes/s, respec- tively (Fig. $5 a$, black lines, inset). The mean and SD of the discharge rate for the target neurons were 253.00 and 124.23 spikes/s, respectively (Fig. $5 a$, gray bars, inset).

When four stimuli appeared and monkey w performed with $>75 \%$ accuracy the mean and SD of the discharge rates for distractor neurons were 216.57 spikes/s (85.21 spikes/s) (Fig. 5b, inset, black lines). The mean and SD of the discharge rates across all neurons for target neurons when monkey w performed the task with $>75 \%$, were 211.24 and 104.52 spikes/s (Fig. 5b, inset, gray bars). This is a mean $\sim 5$ spikes/s difference between the target and distractor neuronal activity. The median difference was 17.2 spikes/s and, although slight and in the opposite direction, the median differences were statistically significant (Wilcoxon test, $p=0.04)$. In the subset of trials for which monkey w's performance was $<75 \%$ accurate, the mean and SD of the distributions of distractor neuron discharge rates were 261.58 and 98.49 spikes/s, respectively, whereas for the target neurons the mean and SD of the discharge rate distributions were 272.74 and 130.56 spikes/s, respectively. Although the mean difference was $\sim 11$ spikes/s, the median difference was 14.56 spikes/s between target and distractor neuronal activity and in the correct direction (i.e., target $>$ distractor). These differences, however, were not statistically significant (Wilcoxon test, $p=0.25$ ). Nevertheless, the relative difference in activity for the $>75 \%$ accurate trials was greater than the relative difference in activity for the $<75 \%$ accurate trials. The data from this monkey show an extreme case in which the neuronal activities were largely inseparable and performance in the selection task was exceptionally poor. Despite this, the relative differences between target and distractor activity correlated with the level of performance even in this monkey whose performance was overall very poor (51\%).

As a step toward quantifying the discriminability of the target and distractor neuronal activity as it relates to performance in the selection task, we computed the area under the ROC curves and the discriminability index $d^{\prime}$, in the usual manner (see Materials and Methods). Comparing target neuronal activity with the maximum distractor neuronal activity across all the neurons from all monkeys, for the subset of data in which monkeys performed with $>75 \%$ accuracy, revealed a mean ROC area of 0.66 and $d^{\prime}$ of 0.56 (Fig. $6 a$, black curve, inset for monkey w) (ROC area, 0.47; $\left.d^{\prime},-0.31\right)$. The ROC area 0.66 was significantly different from 0.50 (permutation test, $p<0.001$ ). For the subset of data in which performance was $<75 \%$ accurate, the ROC area was 0.57 and $d^{\prime}$ was 0.24 (Fig. $6 a$, gray curve, inset for monkey w) (ROC area, $\left.0.53 ; d^{\prime},-0.02\right)$. This area also was significantly different from 0.50 (permutation test, $p<0.01$ ). The two ROC areas obtained from the $<75 \%$ accuracy condition and the $>75 \%$ accuracy condition were significantly different from one another (permutation test, $p<0.01$ ).

To show the relationship between selection performance and individual SC buildup neurons, we plotted the number of neurons against the ROC area obtained for each neuron in the $>75 \%$ performance accuracy subset of trials (Fig. 6b) and for each neuron in the $<75 \%$ performance accuracy subset of trials (Fig. $6 c$ ). This analysis is equivalent to that of others in which they illustrate the number of neurons having a particular choice probability, also referred to as a sender operating characteristic (Newsome et al., 1989a,b; Britten et al., 1996; Dodd et al., 2001; Mazurek et al., 2003; Uka and DeAngelis, 2004; Purushothaman and Bradley, 2005). We first computed ROC area values for each target neuron on a trial-by-trial basis for all 30 data sets. For the 120 neurons, we obtained an ROC area. These 120 neurons were associated with behavioral performance that was either $>75$ or $<75 \%$ accurate 

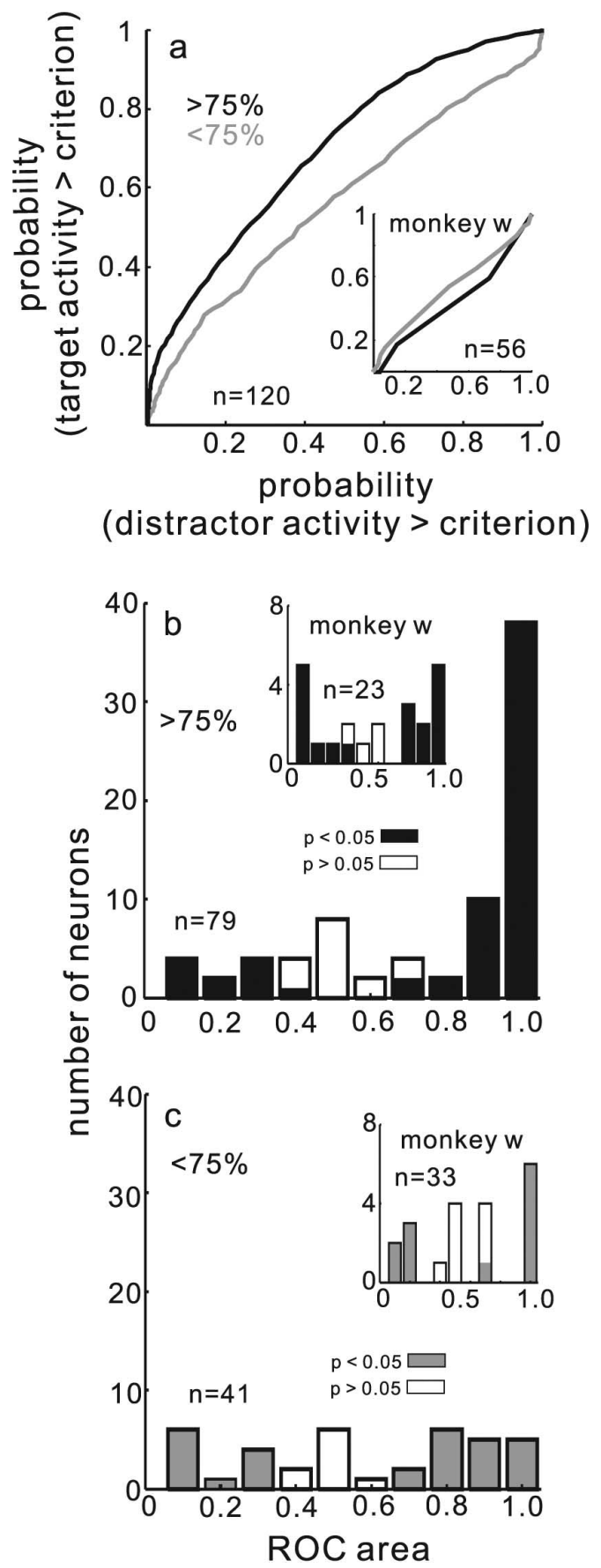

Figure 6. Separability of target and distractor neuronal activity predicts performance. $\boldsymbol{a}, \mathrm{ROC}$ curves computed from the target and the distractor neuronal activity (see Materials and Methods) for data in which performance was $>75 \%$ accurate (black line) and when performance was $<75 \%$ accurate (gray line). $\boldsymbol{b}$, Distribution of ROC areas (CPs) for individual SC neurons measured in the $>75 \%$ correct subset. The filled bars indicate statistically significant ROC areas (permutation test, $p<0.05$ ). The unfilled bars indicate nonsignificant areas. $\boldsymbol{c}$, Same as in $\boldsymbol{b}$ for $<75 \%$ correct subset of data. $n$ in each panel indicates the number of neurons contributing to the plot. Insets in each panel show data for monkey w.

and, therefore, we could divide the neurons into two groups. The range of ROC area values [choice probabilities (CPs)] obtained in the $>75 \%$ accuracy condition varied from low (0.38) to very high (1.0). Of the 79 neurons in the $>75 \%$ accuracy condition, 63 had statistically significant CPs $(74 \%$ permutation test $p<0.05)$ (Fig. $6 b$, filled black bars). Of these, 52 of $63(82.5 \%)$ had CPs $>0.50$. In monkey w, 18 of $23(78.26 \%)$ of the neurons had a statistically significant CP (Fig. 6b, inset filled black bars) (permutation test $p<0.05$ ). Figure $6 c$ illustrates the individual neuron results from the subsets of trials in which performance accuracy was $<75 \%$. Twenty-nine of $41(70.73 \%)$ neurons had a statistically significant CP (permutation test, $p<0.01$ ) (Fig. $6 c$, gray filled bars). Of these, 18 of $29(62 \%)$ had a CP $>0.50$. The inset in Figure $6 c$ illustrates monkey w's results. It is apparent from the illustration that the range of CP (ROC areas) shifts toward higher values as performance accuracy increases. Thus, trial-by-trial variations in performance accuracy are predicted by the relative level of target and distractor neuronal activity in SC buildup neurons. Consistent with an SDT approach to visual search at the level of SC neurons, increased discriminability between target and distractor neuronal activity was associated with better performance. Decreased discriminability between target neuronal activity and distractor neuronal activity was associated with poorer performance.

\section{Discriminability of target and distractor neuronal activity scales with selection accuracy}

One of the influential examples of applying an SDT framework to neuronal activity revealed how neurons in the middle temporal (MT) area of extrastriate cortex signaled the direction of visual motion within a display and how these signals predicted choice accuracy (Newsome et al., 1989a,b; Britten et al., 1992; Parker and Newsome, 1998). ROC analysis showed that the area under the ROC curve determined from MT neuronal activity correlated with the quality of sensory information used for the choice and with choice accuracy. When the motion signal was strong, the ROC area approached 1 and choices were highly accurate (Newsome et al., 1989a,b; Britten et al., 1992).

We applied a similar logic to assess the relationship of the behavior of SC neurons in the four stimulus task to the selection accuracy of the monkeys. It is important to make two points regarding our application of this. First, in other analyses the activity of neurons is compared with the activity of idealized "antineurons" (neurons that are assumed to have an equal and opposite response to a neurons' preferred response). In our analysis, we did not have to make that assumption because we recorded from neurons representing the distractor stimuli directly and at the same time as we recorded neurons representing the target stimulus. Therefore, the comparisons reported here arise from what happens in the brain in real time. Second, in the current analysis, the sensory information on which the selection was made did not vary; monkeys always made a color discrimination to select the target and SC neurons are insensitive to color (Ottes et al., 1987). Therefore, our analysis is most comparable with previous experiments in which CPs were determined on trials in which the same motion coherence appeared (Newsome et al., 1989a,b; Roitman and Shadlen, 2002; Mazurek et al., 2003), or with previous experiments in which CPs were determined when a single stimulus led to two possible percepts, so-called bistable images (Dodd et al., 2001; Krug et al., 2004). Together, we reasoned that if ROC area values scaled with performance accuracy it would indicate that the SC contributes information related to a decision variable rather than signaling purely sensory or motor information (Shadlen and Newsome, 2001; Roitman and Shadlen, 2002).

We pooled all of the trials from monkey $m$ and $c$ (separately pooled monkey w trials), collapsed the correct and error trials together to determine the percentage of total trials, and then sorted all of the data based on the percentage of trials with selection accuracies ranging from 35 to $100 \%$. Note that we only an- 

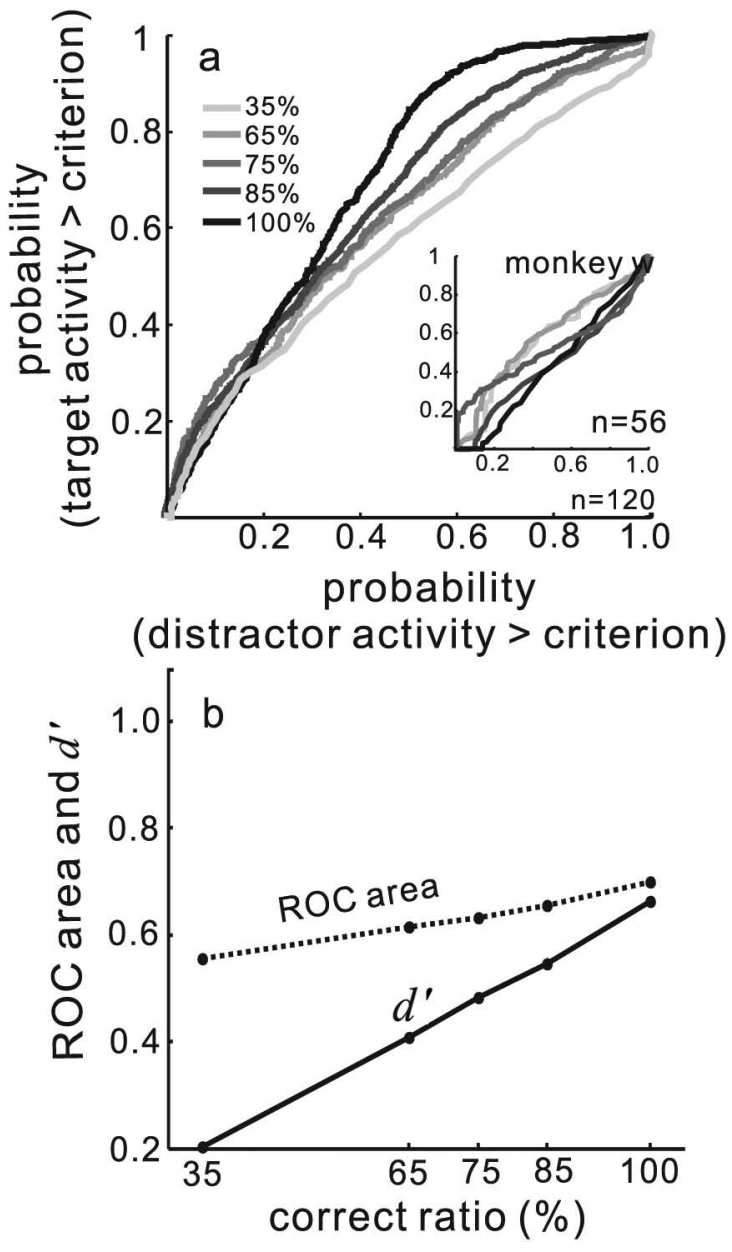

Figure 7. Separation and discriminability of target and distractor neuronal activity scale with performance accuracy. $\boldsymbol{a}$, The entire data set for the two monkeys was sorted by performance. The inset shows the entire data set for monkey w. All trials across all neurons were sorted into subsets of $35,65,75,85$, and $100 \%$ correct trials. ROC curves were computed from the discharge rates of target and distractor neurons as described in Materials and Methods. ROC curves are plotted for the different performance levels and shades of gray indicate performance level. Light gray indicates poorest performance ( $35 \%$ of trials performed correctly), and the black line indicates best performance ( $100 \%$ of trials performed correctly; see the inset legend). $n$ indicates the number of neurons contributing to the plot. $\boldsymbol{b}$. ROC area (dashed line) and $d^{\prime}$ (solid line) are plotted on the ordinate. The abscissa shows the ratio of correct to total trials expressed as a percentage. Each point connected by a line is one ROC area or $d^{\prime}$ obtained for each level of performance accuracy.

alyzed data from correct trials. We only included error trials to determine the total number of trials in each experimental session. We address the activity of neurons on error trials below.

We next computed ROC curves to determine CPs, comparing the target neuronal activity and the distractor neuronal activity. For each set of four neuron recordings we used the activity of the distractor neurons with the highest level of discharge measured $100 \mathrm{~ms}$ before saccade onset (see Materials and Methods). Figure $7 a$ illustrates the result. As selection accuracy increased, so did the separability and discriminability of the target and distractor neuronal activities as measured by the area under the ROC curve and $d^{\prime}$, respectively (Fig. $7 a$, gray to black lines). For the $35 \%$ correct subset, the ROC area was 0.55 and $d^{\prime}$ was 0.20 . For the $65 \%$ subset, the ROC area was 0.62 and $d^{\prime}$ was 0.41 . For the $75 \%$ and $85 \%$ subsets, the ROC areas were 0.63 and 0.66 , respectively, and the $d^{\prime}$ values were 0.48 and 0.55 , respectively. For the trials in which monkeys performed with $100 \%$ accuracy, ROC area was maximal at 0.70 and $d^{\prime}$ was maximal at 0.67 . For monkey $\mathrm{w}$, as shown in the insets of Figure $7 a$, the ROC areas were $0.57,0.51$, $0.45,0.48$, and 0.43 . Whereas the $d^{\prime}$ values were, $0.05,0.33,0.21$, -0.31 , and -0.43 for the $35,65,75,85$ and $100 \%$ conditions, respectively. Figure $7 b$ shows a direct comparison of $d^{\prime}$, ROC, and performance accuracy to illustrate the relationship. As the correct ratio increased from $35 \%$ to $100 \%, d^{\prime}$ increased from 0.20 to 0.67 (Fig. $7 b$, solid line) whereas the ROC area (CP) increased from 0.55 to 0.70 (Fig. $7 b$, dashed line). Note that for monkey w, whose performance overall was poor (51\%), there was little to no scaling of the discriminability between target and distractor neuronal activity with performance (Fig. $7 a$, inset).

To determine whether the relationships between the ROC area values, $d^{\prime}$ values, and the correct ratio were statistically significant, we sampled the subsets of data for each of the accuracy conditions and computed the ROC area 100 times. We then determined the slopes of each of the relationships to obtain a distribution of slopes. One distribution was obtained for the $d^{\prime}$ to correct ratio relationship and one distribution was obtained for the ROC area to correct ratio relationship. Comparing the actual slopes (obtained using the method of least squares) to the permuted slopes revealed slopes that were significantly different from 0 in both cases (Fig. 7c) (ROC, $r=0.97, p<0.01$; $d^{\prime}, r=$ $0.96, p<0.01)$.

\section{Discriminability scaling with performance accuracy is independent of saccade characteristics}

Because we measured the discharge of neurons that included activity close to the initiation of the saccade ( $100 \mathrm{~ms}$ before saccade onset) we explored two other possible explanations for the scaling of discriminability between target and distractor neuronal activity with performance accuracy. First, does discriminability scale with saccade target amplitude? One possibility is that there is a systematic difference in the level of discharge for target and distractor neurons when the distance of the target relative to the fixation point varies. To explore this possibility, we resorted the data by the distance of the target from the fixation point and recalculated the ROC curves. Figure $8 a$ shows the result. The ROC area values had a range that was similar to the range obtained when the data were sorted by accuracy, as expected (0.580.72 ). These values, however, did not scale with increasing target distance as would be expected if discriminability between target and distractor neuronal activity depended on target distance. The lack of relationship is further evident from the plot in Figure $8 c$. ROC area and distance from the fixation point were uncorrelated (Fig. $8 c$, dashed line) $(r=0.13 ; p=0.84)$. Similarly, $d^{\prime}$ and distance of the target from the fixation point were uncorrelated (Fig. $8 c$, solid line) $(r=0.21 ; p=0.73)$.

The second possibility we explored was whether disciminability between target and distractor neuronal activity scaled with saccade velocity. It is possible for example that saccade velocity decreased systematically with poor accuracy. There is some evidence that the level of SC discharge is associated with saccade velocity (Edelman and Keller, 1998). Therefore, if the scaling of ROC area with performance resulted from systematic variations in saccade velocity, we should see a similar relationship between ROC area (and $d^{\prime}$ ) and saccade velocity as we found for ROC area and performance accuracy. To explore this, we resorted the data based on saccade velocity and recalculated the ROC curves (Fig. $8 b, d)$. As obtained for the amplitude analysis, the range of ROC areas was similar (0.55-0.69), but ROC area did not scale with saccade velocity. This was most clear when the ROC area and $d^{\prime}$ values were plotted against the peak velocities of the saccades 
(Fig. $8 d$ ). The ROC area showed no correlation with peak velocity (Fig. $8 d$, dashed lines) $(r=0.34 ; p=0.96) . d^{\prime}$ also showed no relationship with peak velocity (Fig. $8 d$, solid lines) $(r=0.15 ; p=0.98)$. Based on these findings, we conclude that the separation and discriminability of target and distractor neuronal activities in SC scales with saccade selection accuracy. The relationship cannot be explained by parameters of the saccades nor can it be explained by the sensory information leading to selection because SC neurons are insensitive to color. Therefore, we conclude that the activity within the SC signals the saccadic eye movement decision.

Discriminability of target and distractor neuronal activity evolves over time

Previous work in SC manipulating saccade target probability showed that the delay period of SC buildup neurons was modulated by probability. The neuronal activity immediately before the saccade in contrast, appeared identical regardless of the probability (Basso and Wurtz, 1997, 1998; Dorris and Munoz, 1998). More recently, saccade-related activity within the FEF movement neurons was reported to scale with task difficulty (Thompson et al., 2005). This result combined with the results shown in Figure 7 led us to ask whether the relationship between performance accuracy and target and distractor neuronal disciminability would remain or disappear as saccade onset approached. If the activity immediately before the saccade is a movement command, discriminability should not scale with performance and therefore, there should be no relationship between ROC area (CP), $d^{\prime}$, and correct ratio. If, however, the activity of buildup neurons immediately before the saccade signals the decision, the scaling of the relationship between correct ratio and ROC area (CP) as well as $d^{\prime}$ should remain.

We performed the same ROC analysis as described above but across multiple time epochs: 100, 80, 60, 40, and $20 \mathrm{~ms}$ before the initiation of the saccade (Fig. 9). At $100 \mathrm{~ms}$ before the onset of a saccade, the ROC area scaled linearly with performance accuracy (Fig. $9 a$, blue dashed lines) as shown as a black dashed line in Figure $7 b$. As the discharge rate measurement interval approached the time immediately before the saccade $(-20-0 \mathrm{~ms})$, ROC area continued to scale with performance accuracy, although not as steeply (Fig. $9 a$, solid black line). Note also that regardless of performance accuracy, the ROC area at this time was larger than that measured $100 \mathrm{~ms}$ before the saccade (Fig. 9a, compare blue dashed line, solid black line).

To appreciate the change in the relationship between the ROC area and performance accuracy over time, we normalized the data by subtracting the ROC area obtained in the 35\% accuracy condition from all the other accuracy conditions to obtain what is effectively a fractional change in $\mathrm{CP}$ with performance accuracy (Fig. 9b). For the $100 \mathrm{~ms}$ epoch, the area under the ROC curve increased by $14 \%$ when performance accuracy increased from 35 to $100 \%$ (Fig. $9 b$, blue line). For the $80 \mathrm{~ms}$ epoch, the area under the ROC curve also increased by $14 \%$ when performance accuracy increased from 35 to $100 \%$ (Fig. $9 b$, light gray line). For the $60 \mathrm{~ms}$ epoch, the area under the ROC curve increased by $12 \%$ (Fig. $9 b$, gray line). For the $40 \mathrm{~ms}$ epoch, the area under the ROC curve increased by $9 \%$ (Fig. $9 b$, dark gray line), and for the $20 \mathrm{~ms}$ epoch, the area under the ROC curve increased by $7 \%$ when performance accuracy increased from 35 to $100 \%$ (Fig. $9 b$, black line). Thus, the relationship between ROC area and performance accuracy remained even $20 \mathrm{~ms}$ before saccade initiation, although it was not as strong as earlier in time.

Quantifying the discriminability of the probability distributions using $d^{\prime}$ revealed the same trend (Fig. 9c). As performance accuracy increased, $d^{\prime}$ also increased. Furthermore, $d^{\prime}$ scaled with performance accuracy even $20 \mathrm{~ms}$ before saccade onset, although not as steeply as seen for the interval $100 \mathrm{~ms}$ before the saccade. As noted for the ROC area, by $20 \mathrm{~ms}$ before saccade onset, $d^{\prime}$ was maximal across all accuracy conditions (Fig. $9 c$, compare blue and black lines).

Given that we found a relationship between performance accuracy and target and distractor neuronal discriminability even $20 \mathrm{~ms}$ before the onset of a saccade, we concluded that this activity was not a signal for a movement command but rather signaled the saccade decision. Figure 9 shows the relationship between ROC area and $d^{\prime}$ and correct ratio as the time of saccade initiation approached. Note, however, that the duration of the time over which we measured the discharge was longer for the $-100 \mathrm{~ms}$ 

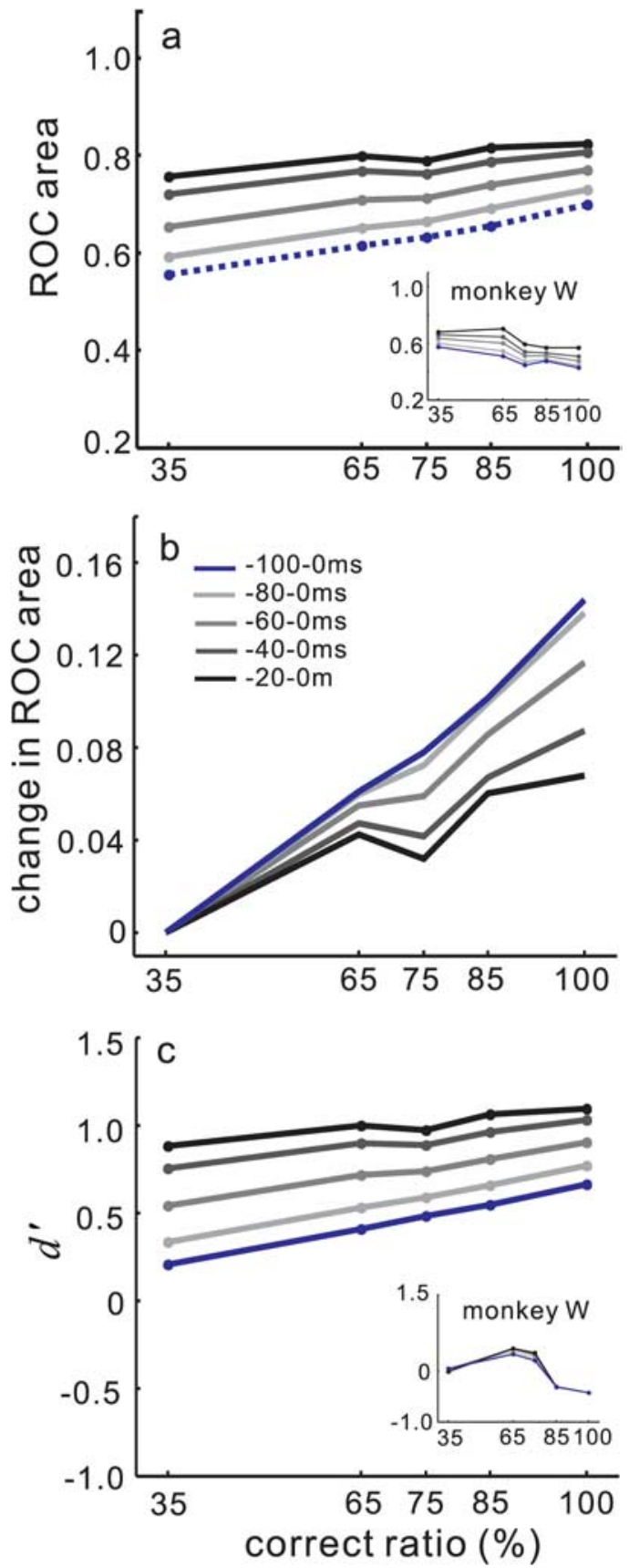

Figure 9. Dynamics of the separation and discriminability of target and distractor neuronal activity as the saccade evolves. $\boldsymbol{a}$, ROC area computed from target and distractor neuronal activity is plotted against the percentage of correct trials. The dashed blue line is the same as the black dashed line shown in Figure $7 b$ in which the ROC area was computed using the $100 \mathrm{~ms}$ interval preceding saccade onset. The black line shows the ROC areas obtained when the discharge epoch used was $20 \mathrm{~ms}$ before saccade onset. The lines increase in lightness up to the blue line, as the measurement interval includes more time before the saccade onset. The line shading scale is shown in the legend in $\boldsymbol{b} . \boldsymbol{b}$ shows the normalized ROC area plotted against performance accuracy level as a percentage of correct trials. The same data as shown in $\boldsymbol{a}$ were normalized by subtracting the $35 \%$ correct ROC area from each of the ROC areas in the different accuracy levels. This resulted in all the first points beginning at $0 . c, d^{\prime}$ plotted against performance accuracy. Each point connected by a line is a $d^{\prime}$ value computed for each accuracy level. The solid blue line is the same line as that shown in Figure $7 b$ as a black line. The line shading legend in $\boldsymbol{b}$ applies.

condition and the $-20 \mathrm{~ms}$ condition. Because this analysis manipulated both the time and the length of the epoch used, we performed a second analysis using $20 \mathrm{~ms}$ duration epochs for each of the five time points to quantify the discharge in target and distractor neurons. Figure $10 a$ shows the relationship between the area under the ROC curve and performance accuracy for five different $20 \mathrm{~ms}$ measurement epochs. When performance accuracy increased from 35 to $100 \%$, the ROC area at $100 \mathrm{~ms}$ before the onset of the saccade ranged from 0.31 to 0.36 , a $5 \%$ increase in ROC area (Fig. 10a,b, light gray line). At $80 \mathrm{~ms}$ before the onset of the saccade, ROC area ranged from 0.31 to 0.41 , a $10 \%$ increase in area (Fig. 10a,b, medium gray line). At $60 \mathrm{~ms}$ before saccade onset, the ROC area ranged from 0.40 to 0.57 , a $17 \%$ increase in ROC area (Fig. 10a,b, gray line). By $40 \mathrm{~ms}$ before saccade onset the ROC area increased from 0.60 to 0.77 when performance increased from 35 to $100 \%$ accurate (Fig. 10a, darkest gray line). This also represents a $17 \%$ increase in ROC area (Fig. 10b, darkest gray line). Interestingly, at $20 \mathrm{~ms}$ before saccade onset, ROC area increased from 0.79 to 0.89 as accuracy increased from 35 to $100 \%$ (Fig. 10a, black line). This is a $10 \%$ increase in ROC (Fig. $10 b$, black line).

Using the same bootstrapping procedure as used to analyze the results shown in Figure 8, we sampled the data to generate distributions of slopes for the ROC area values computed for each of the five measurement epochs, 100-80, 80-60, 60-40, 40-20, and $20-0 \mathrm{~ms}$ before the initiation of the saccade. We then performed a one-way ANOVA to compare the slopes across the different measurement epochs. This analysis revealed a statistically significant main effect (Fig. 10b) (ANOVA, $F_{(4,499)}=$ 716.42; $p<0.001$ ). Post hoc paired comparisons (TukeyKramer) revealed that the $20 \mathrm{~ms}$ epochs beginning at $80 \mathrm{~ms}$ and $60 \mathrm{~ms}$ differed significantly from the $20 \mathrm{~ms}$ epoch beginning at $100 \mathrm{~ms}$. The $20 \mathrm{~ms}$ epochs beginning at 40 and $20 \mathrm{~ms}$ differed significantly from the $100 \mathrm{~ms}$ epoch. Together these results lead to interesting conclusions. First, the largest scaling of ROC area (CP) relative to performance accuracy occurs between 40 and 80 $\mathrm{ms}$ before saccade initiation. Second, earlier than $80 \mathrm{~ms}$ before saccade onset, there is a much smaller relationship between ROC area (CP) and performance accuracy. Likewise, within $40 \mathrm{~ms}$ of the saccade onset, the relationship between ROC area and performance is again diminished. Together we conclude that SC activity maximally signals the saccade decision only transiently - from 80 to $40 \mathrm{~ms}$ before saccade onset. Furthermore, because we observed robust scaling of ROC area and performance accuracy for 80-60 $\mathrm{ms}$ and 60-40 ms before the saccade was initiation, we conclude that the minimum time needed to integrate the discharge from target and distractor neurons to predict saccade choice in this task is at least $20 \mathrm{~ms}$.

An additional way we explored the dynamics of the discriminability between the target and distractor neuronal activity was to perform the ROC analysis on a millisecond by millisecond basis beginning at the time of array onset and lasting for $300 \mathrm{~ms}$. Because this is a common procedure for analyses performed by others, we could also compare our results with those published previously for FEF (Thompson et al., 1996, 2005), SC (McPeek and Keller, 2002) and LIP (Thomas and Paré, 2007). We performed the ROC analysis on data sorted by performance ( $>75 \%$ accurate and $<75 \%$ accurate) as we had done for previous analyses also to determine whether the time course varied with selection accuracy.

Figure 11 illustrates the result of the ROC analysis performed over time. When the array appeared, the area under the ROC curve was variable and even smaller than 0.50 indicating that the distractor activity was larger than the target activity initially. Over time as saccade selection evolved, the ROC area increased and reached the arbitrary, 0.75 area by $125 \mathrm{~ms}$ when performance accuracy was $>75 \%$ (Fig. 11, black line). The same trend was 

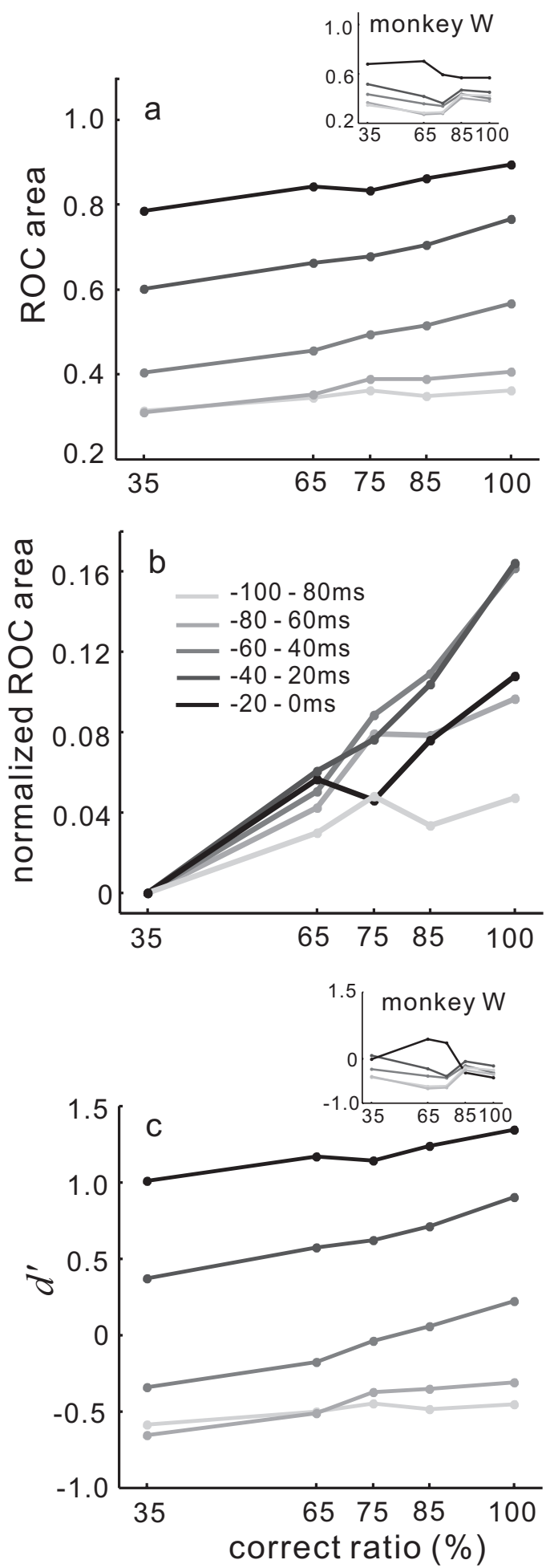

Figure 10. Separation and discriminability of target and distractor neuronal activity increases as the saccade evolves. $\boldsymbol{a}, \mathrm{ROC}$ area computed in $20 \mathrm{~ms}$ epochs at different time intervals as the saccade evolves and plotted against performance accuracy. Each point connected by a line is the ROC area computed for the particular performance accuracy expressed as a percentage of correct to total trials. The lightest gray line shows the ROC area values computed for the $20 \mathrm{~ms}$ interval beginning at -100 and ending at -80 ms before saccade onset. Shades of gray increasing in darkness to the black line indicate $20 \mathrm{~ms}$ epochs moving closer to saccade onset. The black points and line is $20 \mathrm{~ms}$ before saccade onset. Data for monkey $w$ are shown as an inset. The line shading key is shown in $\boldsymbol{b} . \boldsymbol{b}$, The same data as shown in $\boldsymbol{a}$ are normalized in the same way as in Figure $9 b$ and plotted against performance accuracy. $c, d^{\prime}$ for the same $20 \mathrm{~ms}$ epochs is plotted against performance accuracy expressed as a percentage. The scale in $\boldsymbol{b}$ applies. Note that the low values of ROC area early in the trial indicate that one of the three distractor neurons had higher activity than the target neurons at this time. observed on trials when performance was less accurate. Discriminability between target and distractor neuronal activity took longer to evolve: $149 \mathrm{~ms}$ after the array appeared (Fig. 11, gray line) when performance was less accurate. This is consistent with the hypothesis that monkeys found these trials more difficult. Furthermore, the times of discrimination we report here are consistent with the reports of others in SC (McPeek and Keller, 2002) and other saccade-related areas such as FEF (Thompson et al., 1996) and LIP (Thomas and Paré, 2007).

To determine whether the time of neuronal discrimination predicted the time of the saccade, for each neuron, we determined the time at which the ROC area value reached an arbitrary area of 0.75 (McPeek and Keller, 2002). Neurons that did not reach the 0.75 area criterion were excluded. Therefore, only 55 of 120 neurons are illustrated for the $>75 \%$ accuracy condition and 19 of 120 neurons are illustrated for the $<75 \%$ accuracy condition. For the same trials we measured the latency of the saccade. We then sorted the trials into three saccade latency ranges and two performance conditions ( $>75 \%$, short, 108.3-173.9 ms; medium, 118.8-191.1 ms; and long, $137.8-222.3 \mathrm{~ms} ;<75 \%$, short, 132.8-194.4 ms; medium, 144.2-229.3 ms; and long, 154.6-243 $\mathrm{ms})$. A plot of the neuronal discrimination time against saccade latency is shown in Figure $11 b$. Virtually all neurons had a linearly increasing relationship between discrimination time and latency indicating that neuronal activity predicted the time of the saccadic eye movement. Indeed, although the saccade latency was shifted systematically toward longer times, the same trend occurred for the $>75 \%$ correct (Fig. 11b, black lines) and the $<75 \%$ correct trials (Fig. 11b, gray lines). Figure $11 c$ shows the distribution of slopes obtained for all of the data shown in Fig. $11 b$.

\section{Discriminability and errors of selection}

As indicated thus far, the behavior of the monkeys in the selection task was variable. That allowed us to explore the relationship between variations in activity among SC neurons representing targets and distractors and variations in selection accuracy. We found that as performance accuracy increased, the discriminability between target and distractor neurons also increased. Similarly when performance was poor, discriminability between target and distractor neurons was poor. The discriminability between target and distractor neuronal activity took time to evolve once the selection array appeared. The evolution of discriminability, as measured by the time course of the area under the ROC curve, was faster when performance was better. Together these results indicate that the activity of SC buildup neurons carries more information than simple movement commands. This conclusion is in line with previous evidence suggesting that SC neurons encode the location of a target as a movement goal, independent of the movement (Basso et al., 2000; Krauzlis and Dill, 2002; McPeek and Keller, 2002; Port and Wurtz, 2003). Although we do not think it is useful to argue whether these signals are "sensory or motor," we do think it is fruitful to distinguish whether SC neurons signal something higher order like a decision variable (Shadlen and Newsome, 2001; McPeek and Keller, 2002, 2004; Mazurek et al., 2003; Ratcliff et al., 2003, 2007). To address this, we reasoned that if SC neurons signaled the monkeys' decision about the saccade rather than the saccade itself or the stimulus itself, then the relative activity of neurons representing the saccade endpoints (regardless of whether they were experimentally defined as targets or distractors) and neurons representing the other possible locations should lie somewhere between the $>75$ and $<75 \%$ accuracy conditions. In this way, the activity would indicate the level of 

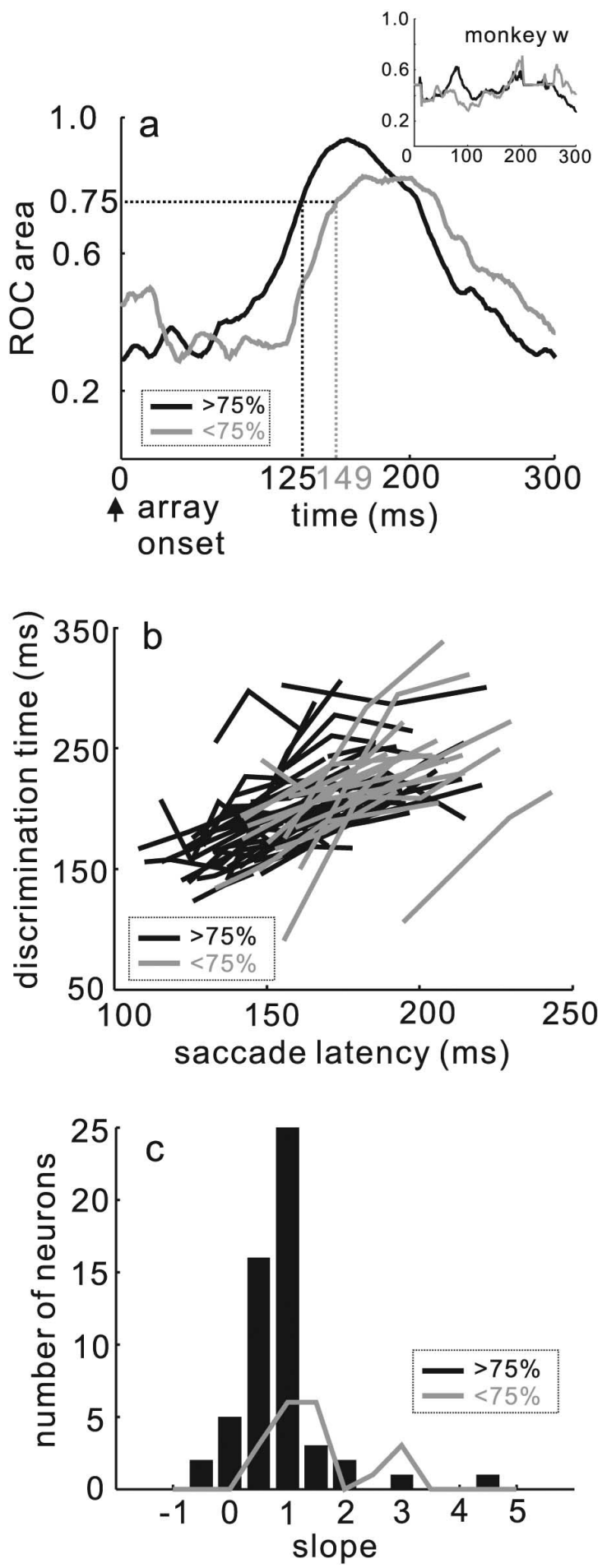

Figure 11. The time course of the evolution of target and distractor neuron separability correlates with performance accuracy and predicts saccade latency. $\boldsymbol{a}, \mathrm{ROC}$ area plotted against time in milliseconds beginning when the stimulus array appears (see Materials and Methods). The black line shows the ROC area calculated for the data when the monkeys' performance was $>75 \%$ accurate. The gray line shows the ROC area when the monkeys' performance was $<75 \%$ accurate. The dashed vertical lines indicate the time point when the ROC area reached 0.75 . The inset shows the same for the data from monkey w. $\boldsymbol{b}$, Discrimination time in milliseconds plotted against saccade latency in milliseconds. Discrimination time is the time point when the $\mathrm{ROC}$ area reached 0.75 . The gray lines show the relationship for the trials in which monkeys performed with an accuracy level $<75 \%$ correct. The black lines show the relationship for the trials in which monkeys performance with an accuracy level $>75 \%$ correct. c, The slopes of the regression lines describing the relationship between discrimination time and saccade latency (see Results) are plotted. The black bars are the data from the $>75 \%$ accuracy subset. The gray distribution shows the data from the $<75 \%$ accuracy subset. confidence monkeys had regarding their decision. If, however, the activity signals the movement output then the neuronal activity on error and correct trials should be indistinguishable.

Up to now we have been referring to neurons as target neurons based on the cue location. For the correct trials the cue and saccade locations are the same. For error trials the cue and the saccade locations are different. So, we will now refer to target neurons as those that either contain the cue (correct) or indicate the saccade location (errors). Figure 12a shows the comparisons of the target neuronal discharge rates obtained in the correct $(>75 \%$ accuracy, black lines) and the error (0\% accuracy, cyan lines) trials. The mean level of discharge in target neurons in correct trials was 262.07 spikes/s, whereas the mean level of discharge in target neurons for error trials was 263.24 spikes/s. Comparing the medians of each distribution indicated that the differences were not statistically significant (Wilcoxon test, $p=0.93$ ) (Fig. 12a, compare thick black and cyan lines). If we left the analysis at this point, we would conclude that the neuronal activity predicts the movement, because the activity was the same regardless of the cue that instructed the movement. Comparing the level of activity from the other neurons, however (distractors in correct trials and nonselected target or distractors in error trials), revealed that the mean level of discharge was 183.90 spikes/s in $>75 \%$ correct trials and 199.34 spikes/s in error trials. The median differences were statistically significant (Wilcoxon test, $p<0.01$ ) (Fig. 12a, thin cyan lines are shifted slightly rightward of the thin black lines). This difference is better appreciated by the illustration shown in Figure 12c. The mean discharge rate for target neurons in $>75 \%$ correct and error trials was similar (Fig. 12c, compare left black and cyan dots). The mean discharge rate for the distractors between $>75 \%$ and error trials was different (Fig. 12c, right black and cyan dots). This result indicates that the target neuronal activity was similar, but the distractor activity was reduced in $>75 \%$ correct trials compared with error trials. In other words, greater suppression of distractor activity was associated with better performance.

Performing the same comparisons, but now for the trials in which performance accuracy was $<75 \%$, showed a different trend. The mean level of discharge in the target neurons in correct trials was 241.24 spikes/s, whereas in error trials the mean level was 263.24 spikes/s. Comparing the medians of each distribution indicated that the differences were statistically significant (Wilcoxon test, $p<0.01$ ) (Fig. 12b, compare thick orange and cyan lines). This difference suggests that the target neuronal activity may signal some level of confidence the monkey has in its decision. Interestingly, the level of activity from the other neurons (distractors in correct trials and nonselected target or distractors from the error trials) revealed that the mean level of discharge was 217.13 spikes/s in $<75 \%$ accuracy trials and 199.34 spikes/s in error trials. The median differences were statistically significant (Wilcoxon test, $p<0.01$ ) (Fig. 12b, thin orange lines are shifted slightly rightward of the cyan lines). Again this interaction is better appreciated by the illustration in Figure 12c. Comparing the orange and cyan dots shows that the target activity was smaller in the $<75 \%$ correct trials compared with error trials and the distractor activity was greater in the $<75 \%$ correct trials compared with error trials (Fig. 12c). In other words, both a reduction of target activity and an enhancement of distractor activity were associated with poor performance, but not with errors. It is as if the relative level of target and distractor neuron activity reveals the level of confidence the monkeys have in their saccade decision. This subtle but significant difference would be impossible to observe with single-electrode recordings. 
If the relative level of activity between target and distractor neurons tells us something about the confidence monkeys had regarding their decision, we predicted that ROC area between correct and error trials should scale in a manner similar to what we observed in Figure 7. It was possible however that the ROC area for error trials would be even less that that obtained for the very poor performance (recall the $35 \%$ accuracy condition had an ROC area of 0.55). Based on the results shown in Figure $12 a-c$, we suspected that the monkeys had some level of confidence in their decision despite what the task instructed. Therefore, we expected to see the ROC area for error trials lie in between the accurate and poor performance.

Figure $12 d$ illustrates the result of the ROC analysis when the data were sorted into error and correct trials, both $>75$ and $<75 \%$ accuracy conditions. The orange and black lines shown for the $>75$ and $<75 \%$ accuracy conditions are the same as those shown in Figure $6 a$ as gray and black lines. The cyan line shows the ROC result for the error trials. The ROC area for these data was 0.65 . Recall that the ROC area for the $>75 \%$ trials was 0.66 and for the $<75 \%$ trials was 0.57 . Each ROC area differed significantly from each other (permutation test and ANOVA, $F_{(2,2999)}=$ 42320.77, $p<0.001$, post hoc TukeyKramer). Monkey w, whose performance overall was very poor, had no such relationship (error trial ROC area, 0.34). Therefore, we conclude that the relative activity of target and distractor neurons within the SC signals not only the saccade decision but the level of confidence monkeys had in that decision.

\section{Discussion}

In this study, we described the results of an experiment in which we recorded from four SC neurons simultaneously while monkeys performed a simple, oddball selection task (Schall, 1995; McPeek and Keller, 2002). We found that when the discriminability between target and distractor neuronal activity was high, selection was likely to be accurate. When the discriminability between the target and distractor neuronal activity was reduced, performance was likely to be poor. Our results yield some general conclusions. First, the combined activity of target and distractor neurons contributes to the selection of a saccade. Second, the relative level of buildup neuronal activity signals the saccade choice and not the saccade characteristics. This relationship between target and distractor neuronal activity and performance accuracy held true even immediately before the saccade onset, precluding an exclusively movement role for this neuronal activity. Importantly, we recorded the neurons representing targets and distractors at the same time. Therefore, in contrast to previous work, the signals we measured were available to the brain in real time, as the selection occurred. Below, we first discuss the relationship of the present results to those found previously in the monkey w.
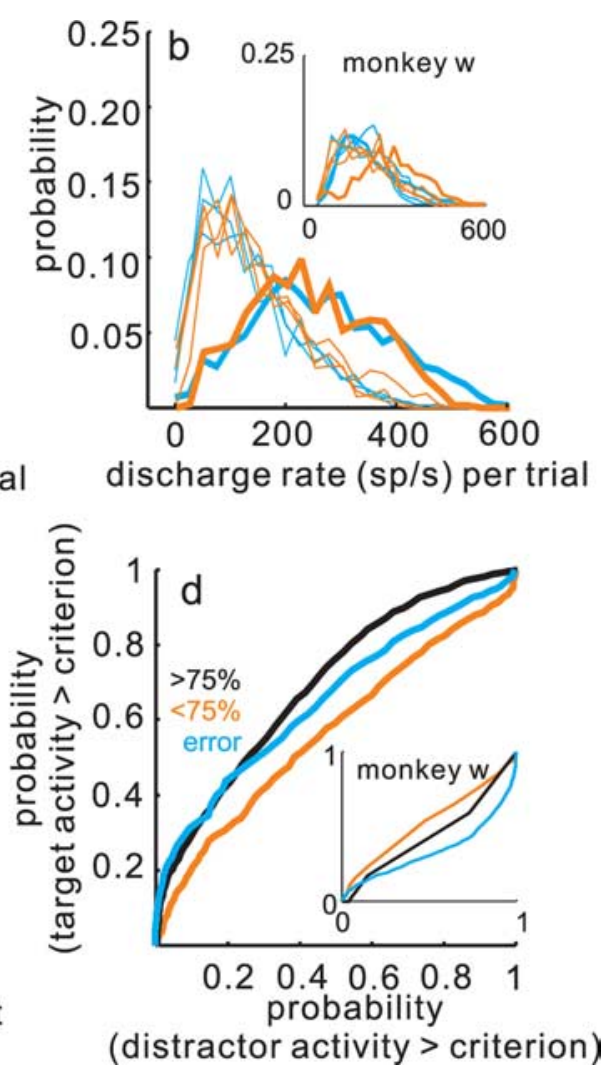

(distractor activity $>$ criterion)

Figure 12. SC buildup activity predicts the confidence of the decision. $\boldsymbol{a}$, The probability of measuring a particular discharge rate in target and distractor neurons in error trials and $>75 \%$ correct trials are superimposed. The black lines show data from the (t) trials when performance was $>75 \%$ accurate and the cyan lines show the data from the error trials. The thick lines are from a distractor neurons in error trials and $<75 \%$ correct trials are superimposed. The cyan lines are the same as shown in a. The orange lines show the data for the $<75 \%$ accurate trials. Thin orange lines are from distractor neurons and thick orange line 列 points are from error trials and orange points are from $<75 \%$ correct trials and black points are from $>75 \%$ correct trials. $\boldsymbol{d}$, ROC curves plotted for $>75 \%$ accurate trials (black), $<75 \%$ accurate trials (orange) and error trials (cyan). The inset shows data from

SC and FEF. Then, we discuss our results in light of current views of the SC in selection and decision making.

\section{Relationship to previous studies in the SC}

Previous experiments in the SC in which the probability of a particular target was manipulated and delay-period activity was measured showed that the activity of buildup/prelude neurons scaled with the probability of selecting a particular saccade target (Basso and Wurtz, 1997, 1998; Dorris and $\mathrm{Mu}-$ noz, 1998). Importantly, at the time the saccade occurred, the discharge of buildup neurons did not vary. This indicated that by the time of the saccade, the output of SC buildup neurons signaled a movement command. The results described here are in contrast to this. We found that even $20 \mathrm{~ms}$ before the onset of a saccade, the discharge of buildup neurons scaled with performance accuracy (Figs. 7, 9, 10). One possible explanation for the difference may be related to the differences in task demands between the previous and the current experiments. Previously, long delays (800-1200 ms) were imposed between the time the array appeared, the time the target was identified and the time the cue to move appeared (Basso and Wurtz, 1998). In the current task, there were no delays. As a 
result, monkeys performed with very high levels of accuracy in the previous tasks and with varying levels of performance in the current task. Furthermore, in the previous experiments (Basso and Wurtz, 1998; Dorris and Munoz, 1998), the positions of the stimuli in the display were constrained by the position of only one electrode whereas in the current experiment the stimulus arrangement was constrained by the positions of four electrodes. This led in some cases, to highly asymmetric displays. We suspect that the emphasis on the speed of selection combined with the asymmetric visual display led to greater uncertainty regarding the target location even at the time of the saccade and despite the constant sensory information (color difference). As such, we think that the current results are consistent with the previous results. They show that SC buildup activity signals the certainty of the saccade choice. But they extend this finding to include the activity right up until the time of the saccade. We suspect that given more time the uncertainty would have declined and performance would have improved in the current task. Indeed, a decline in uncertainty over time is consistent with models that rely on the accumulation of evidence (Mazurek et al., 2003; Ratcliff et al., $2003,2007)$. The increase in ROC area as the saccade evolved is consistent with these ideas (Figs. 9, 10).

A second salient finding we report here is that correct target selection depended on the level of discriminability between the target and distractor neuronal activity. This indicates that the relative level of activity among target and distractor neurons determines which saccade is made. This result is reminiscent of a previous finding in the SC using a brightness-discrimination task (Ratcliff et al., 2007). On trials in which the brightness discrimination was easy the activity of SC neurons representing the correct saccade target was high and the activity of the SC neurons representing the incorrect saccade target was low. When the brightness discrimination was difficult, the activity of SC neurons representing the correct saccade target was also high, but the activity of the SC neurons representing the incorrect target was higher than on easy trials. Although they sorted their data based on task difficulty as defined by the experimenter (differences in quality of the sensory information) and not by the performance of the monkeys with identical sensory information as we did here, their result, nevertheless, is similar to what we report.

The results reported here may also shed light on previous experiments in which injections of muscimol (or lidocaine) were introduced into small regions of SC while monkeys performed the same task as used here (McPeek and Keller, 2004). When a single target appeared in the region of the visual field represented by an inactivated region of SC, saccade accuracy remained intact, albeit saccades occurred with longer latencies (McPeek and Keller, 2004). When the target appeared in the same location, but in the presence of distractors, monkeys made saccades more often to the distractors. Based on our results, we believe we can interpret the muscimol results in an SDT framework. In the presence of only one target, there is only one region of SC active. The mean level of activity would be reduced in the presence of muscimol, but would remain highly discriminable from the activity of the rest of the SC. When multiple possible targets appear, there are multiple points of activation across the SC map. A focal muscimol injection would reduce the mean level (and perhaps the variability) of activity at the injection site thereby altering the disciminability between the target and distractor neuronal activities. As we show here, the reduced discriminability would lead to errors of saccade selection.

\section{Relationship to previous studies in the FEF}

In a task in which monkeys searched for a target that varied along two dimensions, color and shape, recordings in the FEF revealed that the level of activity in distractor neurons was higher when the distractor items were more similar to the target items compared with when the distractor items were less similar to the target. This difference in distractor activity correlated with the difficulty level of the task as well as the performance (Bichot and Schall, 1999; Bichot et al., 2001; Thompson et al., 2005). This result is very similar to what we observed in the SC, although again, they manipulated the sensory information and we did not. Nevertheless, the results suggest that in both the FEF and SC, as the disciminability between target and distractor neuronal populations increases, selection is more likely to be accurate. Furthermore, the activity associated with distractors in FEF movement neurons occurring immediately before a saccade $(-30 \mathrm{~ms})$ was higher in difficult compared with easy trials (Thompson et al., 2005). This was as surprising a result in FEF movement neurons as it is here in SC buildup neurons. First, the movement neurons in FEF and the buildup neurons in SC are likely to be output neurons (Moschovakis et al., 1988a,b; Sommer and Wurtz, 2000; Rodgers et al., 2006). Second, it is generally considered that the activity timelocked to the saccade is the command to initiate a saccade (Sparks, 1975, 1986). Third, neuronal recordings made in the SC and FEF during performance of a task in which the planning of saccades is interrupted occasionally by the appearance of a stop cue (the countermanding task) suggest that neuronal activity immediately before a saccade reaches a fixed threshold which when crossed determines saccade onset (Hanes and Schall, 1996; Paré and Hanes, 2003).

The results we report here (as well as those recently described in FEF) are inconsistent with the fixed threshold hypothesis. Whether these differences reflect difference in task properties as was suggested for FEF (Thompson et al., 2005) remains to be determined. A second possibility is that the activity of buildup neurons is read-out by the saccade-related burst neurons in the $\mathrm{SC}$, which, in turn, rise in activity to a fixed threshold. Although, no difference in the saccade-related discharge of burst and buildup neurons in this regard was obtained in the countermanding task (Paré and Hanes, 2003). We did not record burst neurons and therefore cannot address this point as yet.

\section{The role of SC in decision making}

The results of our experiments are most consistent with a current line of investigation suggesting that decisions based on sensory signals evolve within oculomotor centers of the brain such as LIP, FEF, and SC (Horwitz and Newsome, 1999, 2001; Kim and Shadlen, 1999; Gold and Shadlen, 2000, 2002, 2003; Roitman and Shadlen, 2002; Ratcliff et al., 2003, 2007). We show that the discriminability of target and distractor neuronal activity scales with choice performance (Fig. 7). Importantly, we computed discriminability based on monkeys' choices and not on differences in sensory evidence. We also show that ROC area values or CPs increase as saccade initiation approaches (Figs. 9, 10). This is consistent with findings in the LIP and FEF in which evidence in favor of a decision accumulates over time (Gold and Shadlen, 2007). When we computed discriminability in $20 \mathrm{~ms}$ epochs as the saccade evolved (Fig. 10), we obtained an additional result that, as far as we are aware, has not been reported before in the SC or elsewhere. The maximum change in $\mathrm{CP}$ with performance occurred between 80 and $40 \mathrm{~ms}$ before saccade onset. Later than this time, scaling appeared, but not as steeply (Fig. 10b). We suggest that this change indicates that the rate of accumulation of 
evidence in favor of a saccade decision is a nonstationary process (Ditterich, 2006).

The average ROC area values or CPs we obtained ranged between 0.55 and 0.70 . To compare these results with those reported previously we looked at CPs reported for the motion discrimination task when the motion coherence was $0 \%$. The CPs reported in the FEF (Kim and Shadlen, 1999), LIP (Shadlen and Newsome, 2001), and previously in SC (Horwitz and Newsome, 1999) are very similar to our findings, ranging from 0.60 to 0.75 (Gold and Shadlen, 2001). In area MT/V5 during reports of the perception of a bistable image (Parker et al., 2002; Krug et al., 2004), CPs averaged 0.67. These similar values suggest that the size of the neuronal pools contributing to the judgment in all of these tasks is likely to be similar across these varied brain areas (Britten et al., 1996; Shadlen et al., 1996; Parker et al., 2002). In this light, a first important and unanswered question is how SC buildup neurons are pooled to contribute to a decision. A second important question relates to how the pooled activity is then read-out to produce the saccade. In other words, what is the decision rule? Evidence in FEF and SC suggest that pools of neurons representing a particular action can be considered individual processes racing toward a fixed threshold (Hanes and Schall, 1996; Paré and Hanes, 2003; Boucher et al., 2007). Whichever pool first crosses threshold determines the saccade akin to winner-take-all schemes (Lee et al., 1999). Previous evidence from a brightness discrimination task suggests that the difference between two pools of neurons each representing a possible saccade, is reflected in SC buildup activity that increases to a bound, in a manner similar to a diffusion process (Ratcliff et al., 2003, 2007). Yet another possibility is that neuronal activity is pooled across the entire map of saccades to compute a population vector (Lee et al., 1988; Groh et al., 1997; Groh, 2001). More recent decoding schemes suggest that approaches based on likelihood estimators will hold the key to unlocking decoding strategies (Deneve et al., 1999; Pouget et al., 2003; Ma et al., 2006). Our ongoing investigations using multiple neuron recording are investigating these ideas.

\section{References}

Apter JA (1945) Projection of the retina on superior colliculus of cats. J Neurophysiol 8:123-134.

Basso MA, Wurtz RH (1997) Modulation of neuronal activity by target uncertainty. Nature 389:66-69.

Basso MA, Wurtz RH (1998) Modulation of neuronal activity in superior colliculus by changes in target probability. J Neurosci 18:7519-7534.

Basso MA, Krauzlis RJ, Wurtz RH (2000) Activation and inactivation of rostral superior colliculus neurons during smooth-pursuit eye movements in monkeys. J Neurophysiol 84:892-908.

Bichot NP, Schall JD (1999) Saccade target selection in macaque during feature and conjunction visual search. Vis Neurosci 16:81-89.

Bichot NP, Schall JD (2002) Priming in macaque frontal cortex during popout visual search: feature-based facilitation and location-based inhibition of return. J Neurosci 22:4675-4685.

Bichot NP, Thompson KG, Rao SC, Schall JD (2001) Reliability of macaque frontal eye field neurons signaling saccade targets during visual search. J Neurosci 21:713-725.

Boucher L, Palmeri TJ, Logan GD, Schall JD (2007) Inhibitory control in mind and brain: an interactive race model of countermanding saccades. Psychol Rev 114:376-397.

Bradley A, Skottun BC, Ohzawa I, Sclar G, Freeman R (1987) Visual orientation and spatial frequency discrimination: a comparison of single neurons and behavior. J Neurophysiol 57:755-772.

Britten KH, Shadlen MN, Newsome WT, Movshon JA (1992) The analysis of visual motion: a comparison of neuronal and psychophysical performance. J Neurosci 12:4745-4765.

Britten KH, Newsome WT, Shadlen MN, Celebrini S, Movshon JA (1996) A relationship between behavioral choice and the visual responses of neurons in macaque MT. Vis Neurosci 13:87.

Carello CD, Krauzlis RJ (2004) Manipulating intent: evidence for a causal role of the superior colliculus in target selection. Neuron 43:575-583.

Cohn TE, Green DG, Tanner WJ (1975) Receiver operating characteristic analysis: application to the study of quantum fluctuation effects in optic nerve of Rana pipiens. J Gen Physiol 66:583-616.

Crist CF, Yamasaki DSG, Komatsu H, Wurtz RH (1988) A grid system and a microsyringe for single cell recording. J Neurosci Methods 26:117-122.

Deneve S, Latham PE, Pouget (1999) A Reading population codes: a neural implementation of ideal observers. Nat Neurosci 2:740.

Ditterich J (2006) Evidence for time-variant decision making. Eur J Neurosci 24:3628-3641.

Dodd JV, Krug K, Cumming BG, Parker AJ (2001) Perceptually bistable three-dimensional figures evoke high choice probabilities in cortical area MT. J Neurosci 21:4809-4821.

Dorris MC, Munoz DP (1998) Saccadic probability influences motor preparation signals and time to saccadic initiation. J Neurosci 18:7015-7026.

Edelman JA, Keller EL (1998) Dependence on target configuration of express saccade-related activity in the primate superior colliculus. J Neurophysiol 80:1407-1426.

Egeth HE, Yantis S (1997) Visual attention: control, representation, and time course. Annu Rev Psychol 48:269-297.

Fuchs AF, Robinson DA (1966) A method for measuring horizontal and vertical eye movement chronically in the monkey. J Appl Physiol 21:1068-1070.

Glimcher PW, Sparks DL (1993) Representation of averaging saccades in the superior colliculus of the monkey. Exp Brain Res 95:429-435.

Gold JI, Shadlen MN (2000) Representation of a perceptual decision in developing oculomotor commands. Nature 404:390-394.

Gold JI, Shadlen MN (2001) Neural computations that underlie decisions about sensory stimuli. Trends Cogn Sci 5:10.

Gold JI, Shadlen MN (2002) Banburismus and the brain: decoding the relationship between sensory stimuli, decisions, and reward. Neuron 36:299.

Gold JI, Shadlen MN (2003) The influence of behavioral context on the representation of a perceptual decision in developing oculomotor commands. J Neurosci 23:632.

Gold JI, Shadlen MN (2007) The neural basis of decision making. Annual Rev Neurosci 30:535-574.

Green DM, Swets JA (1966) Signal detection theory and psychophysics. New York: Wiley.

Groh JJ (2001) Converting neural signals from place codes to rate codes. Biol Cybern 85:159-165.

Groh JM, Born RT, Newsome WT (1997) How is a sensory map read Out? Effects of microstimulation in visual area MT on saccades and smooth pursuit eye movements. J Neurosci 17:4312-4330.

Hanes DP, Schall JD (1996) Neural control of voluntary movement initiation. Science 274:427-430.

Hanes DP, Wurtz RH (2001) Interaction of the frontal eye field and superior colliculus for saccade generation. J Neurophysiol 85:804-815.

Hays AV, Richmond BJ, Optican LM (1982) A UNIX-based multiple process system for real-time data acquisition and control. WESCON Conf Proc 2:1-10.

Horwitz GD, Newsome WT (1999) Separate signals for target selection and movement specification in the superior colliculus. Science 284:1158-1161.

Horwitz GD, Newsome WT (2001) Target selection for saccadic eye movements: prelude activity in the superior colliculus during a directiondiscrimination task. J Neurophysiol 86:2548-2558.

Judge SJ, Richmond BJ, Chu FC (1980) Implantation of magnetic search coils for measurement of eye position: an improved method. Vision Res 20:535-538.

Keppel G (1991) Design and analysis: a researcher's handbook, Ed 3. Upper Saddle River, NJ: Prentice-Hall.

Kim JN, Shadlen MN (1999) Neural correlates of a decision in the dorsolateral prefrontal cortex of the macaque. Nat Neurosci 2:176-185.

Krauzlis RJ, Dill N (2002) Neural correlates of target choice for pursuit and saccades in the primate superior colliculus. Neuron 35:355-363.

Krug K, Cumming BG, Parker AJ (2004) Comparing perceptual signals of single V5/MT neurons in two binocular depth tasks. J Neurophysiol 92:1586. 
Lee C, Rohrer WH, Sparks DL (1988) Population coding of saccadic eye movements by neurons in the superior colliculus. Nature 332:357-360.

Lee DK, Itti L, Koch C, Braun J (1999) Attention activates winner-take-all competition among visual filters. Nat Neurosci 2:375-381.

Li X, Basso MA (2005) Competitive stimulus interactions within single response fields of superior colliculus neurons. J Neurosci 25:11357-11373.

Li X, Kim B, Basso MA (2006) Transient pauses in delay-period activity of superior colliculus neurons. J Neurophysiol 95:2252-2264.

Ma WJ, Beck JM, Latham PE, Pouget (2006) A Bayesian inference with probabilistic population codes. Nat Neurosci 9:1432.

Mazurek ME, Roitman JD, Ditterich J, Shadlen MN (2003) A role for neural integrators in perceptual decision making. Cereb Cortex 13:1257.

McPeek RM, Keller EL (2002) Saccade target selection in the superior colliculus during a visual search task. J Neurophysiol 88:2019-2034.

McPeek RM, Keller EL (2004) Deficits in saccade target selection after inactivation of superior colliculus. Nat Neurosci 7:757-763.

Meinecke C (1989) Retinal eccentricity and the detection of targets. Psychol Res 51:107-116.

Moschovakis AK, Karabelas AB, Highstein SM (1988a) Structure-function relationships in the primate superior colliculus. I. Morphological classification of efferent neurons. J Neurophysiol 60:232-262.

Moschovakis AK, Karabelas AB, Highstein SM (1988b) Structure-function relationships in the primate superior colliculus. II. Morphological identification of presaccadic neurons. J Neurophysiol 60:263-302.

Motter BC, Simoni DA (2007) The roles of cortical image separation and size in active visual search performance. J Vision 7:1-15.

Munoz DP, Wurtz RH (1995) Saccade-related activity in monkey superior colliculus. I. Characteristics of burst and buildup cells. J Neurophysiol 73:2313-2333.

Newsome W, Britten K, Movshon A, Shadlen MN (1989a) Single neurons and the perception of visual motion. Neural mechanisms of visual perception. In: Proceedings of the Retina Research Foundation (Lam DK, Gilbert C, eds), pp 171-198. The Woodlands, TX: Portfolio.

Newsome WT, Britten KH, Movshon JA (1989b) Neuronal correlates of a perceptual decision. Nature 341:52-54.

Ottes FP, Van Gisbergen JAM, Eggermont JJ (1987) Collicular involvement in a saccadic colour discrimination task. Exp Brain Res 66:465-478.

Palmer J, Verghese P, Pavel M (2000) The psychophysics of visual search. Vision Res 40:1227-1268.

Paré M, Hanes DP (2003) Controlled movement processing: Superior colliculus activity associated with countermanded saccades. J Neurosci 23:6480-6489.

Paré M, Wurtz RH (2001) Progression in neuronal processing for saccadic eye movements from parietal cortex area LIP to superior colliculus. J Neurophysiol 85:2545-2562.

Parker A, Krug K, Cumming B (2002) Neuronal activity and its links with the perception of multi-stable figures. Philos Trans R Soc Lond B Biol Sci 357:1053-1062.

Parker AJ, Newsome WT (1998) Sense and the single neuron: probing the physiology of perception. Annu Rev Neurosci 21:227-277.

Port NL, Wurtz RH (2003) Sequential activity of simultaneously recorded neurons in the superior colliculus during curved saccades. J Neurophysiol 90:1887-1903.

Pouget A, Dayan P, Zemel RS (2003) Inference and computation with population codes. Annu Rev Neurosci 26:381-410.

Purushothaman G, Bradley DC (2005) Neural population code for fine perceptual decisions in area MT. Nat Neurosci 8:99-106.

Ratcliff R, Cherian A, Segraves M (2003) A comparison of macaque behav- ior and superior colliculus neuronal activity to predictions from models of two choice decisions. J Neurophysiol 90:1392-1407.

Ratcliff R, Hasegawa YT, Hasegawa RP, Smith PL, Segraves MA (2007) Dual diffusion model for single-cell recording data from the superior colliculus in a brightness-discrimination task. J Neurophysiol 97:1756-1774.

Robinson DA (1972) Eye movements evoked by collicular stimulation in the alert monkey. Vis Res 12:1795-1808.

Rodgers CK, Munoz DP, Scott SH, Paré M (2006) Discharge properties of monkey tectoreticular neurons. J Neurophysiol 95:3502-3511.

Roitman JD, Shadlen MN (2002) Response of neurons in the lateral intraparietal area during a combined visual discrimination reaction time task. J Neurosci 22:9475-9489.

Schall JD (1995) Neural basis of saccade target selection. Rev Neurosci 6:63-85.

Schall JD, Hanes DP (1998) Neural mechanisms of selection and control of visually guided eye movements. Neural Netw 11:1241-1251.

Schall JD, Thompson KG (1999) Neural selection and control of visually guided eye movements. Annu Rev Neurosci 22:241-259.

Schall JD, Hanes DP, Thompson KG, King DJ (1995) Saccade target selection in frontal eye field of macaque. I. Visual and premovement activation. J Neurosci 15:6905-6918.

Shadlen MN, Newsome WT (2001) Neural basis of a perceptual decision in the parietal cortex (area LIP) of the rhesus monkey. J Neurophysiol 86:1916-1936.

Shadlen MN, Britten KH, Newsome WT, Movshon JA (1996) A computational analysis of the relationship between neuronal and behavioral responses to visual motion. J Neurosci 16:1486-1510.

Sommer MA, Wurtz RH (2000) Composition and topographic organization of signals sent from the frontal eye field to the superior colliculus. J Neurophysiol 83:1979-2001.

Sparks DL (1975) Response properties of eye movement-related neurons in the monkey superior colliculus. Brain Res 90:147-152.

Sparks DL (1986) Translation of sensory signals into commands for control of saccadic eye movements: role of primate superior colliculus. Physiol Rev 66:118-171.

Thomas NWD, Paré M (2007) Temporal processing of saccade targets in parietal cortex area LIP during visual search. J Neurophysiol 97:942-947.

Thompson KG, Hanes DP, Bichot NP, Schall JD (1996) Perceptual and motor processing stages identified in the activity of macaque frontal eye field neurons during visual search. J Neurophysiol 76:4040-4054.

Thompson KG, Bichot NP, Sato TR (2005) Frontal eye field activity before visual search errors reveals the integration of bottom-up and top-down salience. J Neurophysiol 93:337-351.

Treisman A, Gelade G (1980) A feature-integration theory of attention. Cognit Psychol 12:97-136.

Uka T, DeAngelis GC (2004) Contribution of area MT to stereoscopic depth perception: choice-related response modulations reflect task strategy. Neuron 42:297.

Verghese P (2001) Visual search and attention: a signal detection theory approach. Neuron 31:524-535.

Wolfe J, O'Neill P (1998) Why are there eccentricity effects in visual search? Visual and attentional hypotheses. Percept Psychophys 60:140-156.

Wolfe JM, Horowitz TS (2004) What attributes guide the deployment of visual attention and how do they do it? Nat Rev Neurosci 5:495-501.

Wurtz RH, Goldberg ME (1972) Activity of superior colliculus in behaving monkey: III. Cells discharging before eye movements. J Neurophysiol 35:575-586. 